\title{
The Higgs legacy of the LHC Run I
}

\section{Tyler Corbett, ${ }^{a}$ Oscar J.P. Éboli, ${ }^{b}$ Dorival Gonçalves, ${ }^{c}$ J. Gonzalez-Fraile, ${ }^{d}$ Tilman Plehn ${ }^{d}$ and Michael Rauch ${ }^{e}$}

${ }^{a}$ C.N. Yang Institute for Theoretical Physics, SUNY at Stony Brook, Stony Brook, NY 11794-3840, U.S.A.

${ }^{b}$ Instituto de Fúsica, Universidade de São Paulo, C.P. 66318, 05315-970, São Paulo SP, Brazil

${ }^{c}$ Institute for Particle Physics Phenomenology, Department of Physics, Durham University, Durham, DH1 3LE, U.K.

${ }^{d}$ Institut für Theoretische Physik, Universität Heidelberg, Philosophenweg 16, 69120 Heidelberg, Germany

${ }^{e}$ Institute for Theoretical Physics, Karlsruhe Institute of Technology, Wolfgang-Gaede-Str. 1, 76131 Karlsruhe, Germany

E-mail: corbett.ts@gmail.com, eboli@fma.if.usp.br,

dorival.goncalves@durham.ac.uk, fraile@thphys.uni-heidelberg.de, plehn@uni-heidelberg.de, michael.rauch@kit.edu

ABSTRACT: Based on Run I data we present a comprehensive analysis of Higgs couplings. For the first time this SFitter analysis includes independent tests of the Higgs-gluon and top Yukawa couplings, Higgs decays to invisible particles, and off-shell Higgs measurements. The observed Higgs boson is fully consistent with the Standard Model, both in terms of coupling modifications and effective field theory. Based only on Higgs total rates the results using both approaches are essentially equivalent, with the exception of strong correlations in the parameter space induced by effective operators. These correlations can be controlled through additional experimental input, namely kinematic distributions. Including kinematic distributions the typical Run I reach for weakly interacting new physics now reaches 300 to $500 \mathrm{GeV}$.

Keywords: Higgs Physics, Beyond Standard Model, Effective field theories, Standard Model

ARXIV EPRINT: 1505.05516 


\section{Contents}

1 Introduction $\quad 1$

1.1 Experimental input 2

1.2 Fit setup 4

2 Higgs couplings $\quad 5$

$\begin{array}{lll}2.1 & \text { Standard Model couplings } & 7\end{array}$

$\begin{array}{ll}2.2 \text { Invisible decays } & 10\end{array}$

$\begin{array}{lll}2.3 & \text { Theoretical uncertainties } & 12\end{array}$

3 Higgs operators $\quad 15$

$\begin{array}{ll}3.1 & \text { Dimension-6 operator basis } \\ 3.2 & 16\end{array}$

$\begin{array}{lll}3.2 & \text { Rate-based analysis } & 18\end{array}$

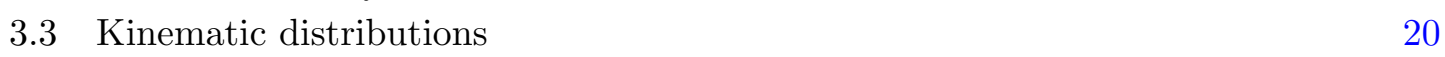

3.4 Full dimension-6 analysis 23

4 Future: off-shell measurements $\quad 25$

$\begin{array}{llr}5 & \text { Present and future } & 28\end{array}$

$\begin{array}{lr}\text { A Numerical results } & 29\end{array}$

\section{Introduction}

The discovery of a light narrow Higgs boson [1-3] in $2012[4,5]$ was a triumph of particle physics. While its statistical significance was largely driven by the peak in the $\gamma \gamma$ invariant mass spectrum, already Run I of the LHC allowed ATLAS and CMS to perform a large number of tests of the nature of the observed resonance. One of these tests is the analysis of Higgs couplings relative to their Standard Model values. No significant deviations from the Standard Model properties were observed in the Higgs production and decay rates. However, we need to keep in mind that these constraints are at a numerical level where in typical weakly interacting models for new physics [6] we would not expect significant deviations, either.

One of the main physics programs defining the upcoming LHC runs will be a comprehensive precision analysis of Higgs properties. It will eventually utilize up to $3000 \mathrm{fb}^{-1}$ of integrated luminosity, covering a wide range of production and decay channels and observables. Technically, the Higgs coupling analysis from Run I is based on comparably simple total cross sections and branching ratios; this simple structure of underlying measurements allows us to limit the interpretation to an independent variation of all Higgs couplings in the Standard Model without missing much of the experimental information. 
Planning for the corresponding Run II analysis we need to implement major extensions to the Higgs coupling analysis. The reason is that the expected wealth of measurements, including kinematic distributions, will probe modifications of the Standard Model Lagrangian which are not captured by a simple shift in Higgs couplings. ${ }^{1}$ Including kinematic distributions in the analysis of the Higgs couplings suggests to describe the theoretical basis in terms of an effective field theory. It also implies that the interface between experiment and theory has to be re-defined. Eventually, at least three questions need to be addressed by the Higgs measurements:

1. do any of the observed Higgs couplings show deviations from their Standard Model predictions?

2. can possible deviations be linked to a mass scale through an effective theory of the electroweak Lagrangian?

3. what does the Higgs sector tell us about specific new physics models?

These questions are closely tied to each other, but the complex structure of correlations between the experimental measurements and between theoretical predictions render a single answer to all three questions unrealistic. In the past, SFITTER has been one of the driving forces to answer the first [7-17] and third [18] questions. The second question has been tackled by Higgs specialized tools, for example presented in refs. [19-30]. A shift from the analysis of Higgs couplings based on total rates to an effective field theory of the Higgs sector is the appropriate step to include kinematic distributions in the Higgs fit.

In this paper we first update the Higgs coupling analysis to include the full Run I data set. One focus of SFITTER is the appropriate treatment of theoretical uncertainties, which will also play a major role in this paper. Second, we present a first SFITTER study of the electroweak effective field theory, which will in the future allow us to treat the three above questions in the same framework. With that purpose we include in the analysis several differential distributions and we study their implementation and impact. The Run I constraints in terms of the accessible new physics scale stay below the $\mathrm{TeV}$ range, which is consistent with a $20 \%$ precision on coupling analyses and supports the statement that within known theoretical frameworks we do not expect to see deviations in the Higgs couplings during Run I. Finally, we include off-shell measurements of Higgs production [31-33] in the SFITTER analysis and estimate their current impact. While it has been established that off-shell measurements will not serve as model-independent measurements of the Higgs width [34-38], we can establish their role in hypothesis-based Higgs studies.

\section{$1.1 \quad$ Experimental input}

In all SFiTTER Higgs coupling analyses the experimental input is not the published set of Higgs signal strengths, but the number of signal and background events for each analysis.

\footnotetext{
${ }^{1}$ This does not mean that the Higgs coupling analysis should be abandoned entirely. For reasons discussed in this paper we will keep it in the SFITTER framework to search for physics beyond the Standard Model which merely shifts Higgs couplings.
} 
This allows us to independently study statistical, systematic, and theoretical uncertainties and include them in a profile likelihood analysis largely independent of the ATLAS and CMS assumptions. As a matter of fact, this independence is our motivation to maintain the SFITTER effort in spite of more and more advanced experimental Higgs coupling analyses.

As experimental input we use the following Higgs searches and measurements as published by the ATLAS and CMS collaborations:

\begin{tabular}{|l|ll|}
\hline production/decay mode & ATLAS & CMS \\
\hline$H \rightarrow W W$ & Ref. [39] & Ref. [40] \\
$H \rightarrow Z Z$ & Ref. [41] & Ref. [42] \\
$H \rightarrow \gamma \gamma$ & Ref. [43] & Ref. [44] \\
$H \rightarrow \tau \bar{\tau}$ & Ref. [45] & Ref. [46] \\
$H \rightarrow b \bar{b}$ & Ref. [47] & Ref. [48] \\
$H \rightarrow Z \gamma$ & Ref. [49] & Ref. [50] \\
$H \rightarrow$ invisible & Ref. [51-54] & Ref. [55, 56] \\
\hline$t \bar{t} H$ production & Ref. [43, 57] & Ref. [44, 58, 59] \\
\hline kinematic distributions & Ref. [47, 60] & \\
\hline off-shell rate & Ref. [61] & Ref. [62] \\
\hline
\end{tabular}

From all these analyses we extract the number of observed, signal and background events after appropriate cuts. The several categorizations in the experimental searches listed in the above table lead to the 159 measurements that we include in the rate based analyses. In section 3.4 we add 14 extra measurements from kinematic distributions. Finally, the off-shell distributions considered in section 4 contribute with 37 additional measurements.

We will show Higgs coupling analyses in the SFITTER framework starting with one universal modification, moving to five tree level SM-like couplings, and then allowing for additional contributions to the Higgs-photon and Higgs-gluon loop-induced couplings. A new channel which we did not include in previous Higgs coupling analyses is the direct measurement of a modified top-quark Yukawa coupling in $t \bar{t} H$ production. This is necessary to identify new physics contributing to the effective Higgs-gluon coupling. While there is not yet enough sensitivity to properly observe the $t \bar{t} H$ production channel with SM coupling strength, the current searches do provide upper bounds on the production and decay rates. Both experiments have looked for this channel as part of their di-photon final-state analysis [43, 44, 57]; moreover, CMS has published additional searches based on multi-lepton final states arising from Higgs decays into $W W^{*}, Z Z^{*}$ and $\tau \bar{\tau}$ [58] as well as a dedicated $b \bar{b}$ analysis [59]. These $t \bar{t} H$ measurements allow us to separate an extra new contribution to the Higgs-gluon coupling, leading to the analysis with seven independent coupling modifications. After setting the two Higgs-weak-boson coupling modifications equal, these seven modifications correspond to the relevant parameters in the non-linear effective Lagrangian expansion [63-67], if we restrict them to the leading terms following ref. [68]. In the last step we will also include Higgs searches to invisible particles, 
i.e. generating missing transverse momentum. This will allow us to add an extra modification to the analysis, accounting for possible Higgs invisible decays. All coupling analyses in the traditional SFITTER framework rely on the same measurements listed above.

For the dimension- 6 operators, which not only change the coupling strengths but also the momentum dependence of the vertices leading to modifications of kinematic distributions, we make use of several differential distributions. These are the transverse momentum distributions of the gauge boson in $V H, H \rightarrow b \bar{b}$ production [47] for all 0,1 and 2-lepton final states, and the $\Delta \phi_{j j}$ distribution in $H+2$ jets with di-photon decays of the Higgs boson [60]. In section 3.3 we will discuss in detail how we include the crucial information from kinematic distributions in addition to total rates.

Finally, we will for the first time include off-shell Higgs production rates [61, 62] in a global fit of Higgs properties. We will analyze their effect to the usual coupling determination and quantify their potential to constrain the Higgs total decay width.

\section{$1.2 \quad$ Fit setup}

The most relevant shortcoming of Higgs production at the LHC is that there is no method which allows us to directly access the Higgs width if we only consider on-shell data. For the traditional Higgs couplings analysis this means that we will be sensitive to all observed Higgs decays, including decays to invisible particles passing through the detector without leaving a trace. Because the total width entering the coupling measurement is a sum of partial widths, we can easily construct a lower limit to the total width by summing the partial widths induced by the observed Higgs couplings.

Once we add off-shell Higgs data we will start being sensitive for example to Higgs decays to hadrons or more than two states. The reason is that off-shell Higgs rate measurements add a possible upper limit on the Higgs width, as discussed in detail in section 4 . This limit is currently weak, so for the present section we identify the sum of observed partial widths with the total width, as usually done in SFitTER. The one additional assumption we need to make is generation universality, meaning that the relation between the mass and the Yukawa coupling for up-type quarks, down-type quarks, and charged leptons is universal for the second and third generations each. For example, the charm Yukawa coupling is shifted by $g_{c}=g_{c}^{\mathrm{SM}}\left(1+\Delta_{t}\right)$ in the usual SFitTer conventions which will be reviewed in eq. (2.1).

With these assumptions we know what would happen if there was a sizeable unobserved contribution to the total width: in the ideal case of all measurements otherwise in agreement with the Standard Model and equally constraining, all measured Higgs couplings based on the underestimate of the total width will appear to be too small by a universal factor. The experimental challenge would be to distinguish such a scenario for example from a Higgs portal where the SM Higgs state mixes with a heavy additional scalar, see e.g. [69-75]. In this situation the off-shell Higgs rate measurement will be extremely useful.

For all experimental uncertainties we assume a Poisson distribution for the statistical error of the rate measurements and Gaussian distributions of associated nuisance parameters. For the systematic uncertainty distribution this is justified as long as it is resolved with help of some kind of measurement outside the analysis we are considering. For theoretical uncertainties a Gaussian modeling might be technically convenient, but it needs to 
be established that it is at least conservative [76]. We will give a detailed discussion of this issue in section 2.3 .

The usual determination of model parameters in SFITTER proceeds in two steps. First, we construct Markov chains which probe the multi-dimensional space of Higgs couplings. These chains cover typical values of $\Delta_{x} \in[-5,7]$. The error treatment includes all experimental and theoretical error bars with full correlations. From these chains we for example compute the 2-dimensional correlations shown later in this work. In a second step we can focus on the SM-like solution, defined as $\Delta_{x}>-1$ for all couplings, and compute the $68 \%$ and 95\% confidence-level (CL) interval of the log-likelihood for each individual Higgs coupling.

In the Higgs analysis presented in this paper we already derive 1-dimensional profile likelihoods and the corresponding $68 \%$ CL interval from the Markov chains. Unlike for the 2-dimensional correlations we limit our $68 \%$ CL analysis in the individual couplings to the SM-like solution. The interval is defined as the range of coupling modifications or Wilson coefficients covering $68 \%$ of the integrated profile log-likelihood. Because in particular for non-Gaussian distributions this definition allows for a simultaneous shift of both limits and is hence not uniquely defined, we also require the value of the profile log-likelihood to coincide on both sides of the error band.

\section{Higgs couplings}

One question we can ask in the Higgs sector is: how well does the Standard Model describe all available Higgs data at the LHC? There are (at least) two ways of answering this question: first, we can compute an over-all confidence level of the Standard Model given all available Higgs data, possibly combined with electroweak precision measurements etc. The answer to this question is statistically well defined, but to give us useful information we need to carefully analyze the pulls of different measurements.

Second, we can measure the parameters in the renormalizable Higgs Lagrangian: we start from the quadratic and quartic terms in the Higgs potential, which can be exchanged for the Higgs mass and the vacuum expectation value $v$. This relation can eventually be tested in measurements of the Higgs self-coupling. In addition, we can measure the Higgs coupling to each Standard Model particle. The Lagrangian underlying this measurement consists of the Standard Model operators with free couplings. These couplings can be extracted from LHC rate measurements,

$$
\begin{aligned}
& g_{x}=g_{x}^{\mathrm{SM}}\left(1+\Delta_{x}\right) \\
& g_{\gamma}=g_{\gamma}^{\mathrm{SM}}\left(1+\Delta_{\gamma}^{\mathrm{SM}}+\Delta_{\gamma}\right) \equiv g_{\gamma}^{\mathrm{SM}}\left(1+\Delta_{\gamma}^{\mathrm{SM}+\mathrm{NP}}\right) \\
& g_{g}=g_{g}^{\mathrm{SM}}\left(1+\Delta_{g}^{\mathrm{SM}}+\Delta_{g}\right) \equiv g_{g}^{\mathrm{SM}}\left(1+\Delta_{g}^{\mathrm{SM}+\mathrm{NP}}\right),
\end{aligned}
$$

where the modifications of the tree-level couplings appearing in the Standard Model loops

are encoded into $\Delta_{\gamma, g}^{\mathrm{SM}}$ while extra possible new physics contributions are included in $\Delta_{\gamma, g}$. In general, the loop-induced couplings have a non-trivial momentum dependence; for the Higgs couplings measurement we assume that all three external momenta are fixed, reducing 
the coupling to a single number. In terms of a Lagrangian we can write our hypothesis as

$$
\begin{aligned}
\mathcal{L}= & \mathcal{L}_{\mathrm{SM}}+\Delta_{W} g m_{W} H W^{\mu} W_{\mu}+\Delta_{Z} \frac{g}{2 c_{w}} m_{Z} H Z^{\mu} Z_{\mu}-\sum_{\tau, b, t} \Delta_{f} \frac{m_{f}}{v} H\left(\bar{f}_{R} f_{L}+\text { h.c. }\right) \\
& +\Delta_{g} F_{G} \frac{H}{v} G_{\mu \nu} G^{\mu \nu}+\Delta_{\gamma} F_{A} \frac{H}{v} A_{\mu \nu} A^{\mu \nu}+\text { invisible decays }
\end{aligned}
$$

with $c_{w}$ denoting the cosine of the weak mixing angle. A possible Higgs decay to invisible states could be described by a wide variety of Lagrangian terms, but as long as we only search for an invisible branching ratio there is no need for further specifications. The contributions to the higher-dimensional Higgs-gluon and Higgs-photon couplings are normalized to their Standard Model values $F_{G}$ and $F_{A}$. We use their values for finite loop masses, while their normalization is illustrated by the limit $F_{G}^{(\infty)} \rightarrow \alpha_{s} /(12 \pi)$ for a heavy top mass. The invisible decay width can for example be generated in a Higgs portal model [69-75] and will be quoted in terms of an invisible branching ratio.

The form $(1+\Delta)$ of the coupling deviations suggests that we will focus on scenarios not too different from the Standard Model. Large deviations from the Standard Model should be taken with a grain of salt, because in such a situation it is not clear if the kinematic distributions of the 'Higgs' signal and the associated detector efficiencies are well controlled. Values around $\Delta=-2$ indicate a switch in the sign of the coupling, which should be checked individually. In some figures we show the full range of $\Delta$ values for illustration, but we will limit our interpretation to small values of $|\Delta| \lesssim 0.5$.

The coupling measurement according to eqs. (2.1) and (2.2) is clearly well defined in the sense that it corresponds to a weak scale Lagrangian which we compare to experimental results. Higher-order QCD corrections can be included, because renormalizability with respect to the strong coupling is not affected by changes in the Higgs couplings. Electroweak corrections cannot be computed in a model with free Higgs couplings, but their impact on LHC rates can safely be neglected for Run I data.

In the spirit of an effective field theory the free couplings ansatz of eq. (2.1) can be linked to extended Higgs sectors [18]: in such models the Higgs coupling measurement will search for modifications to the couplings of the SM-like Higgs boson. Using a well defined ultraviolet completion, possible shifts can also be interpreted for example using the full set of model parameters in an aligned two-Higgs-doublet model. If we include $\Delta_{t}$ as well as $\Delta_{g}$ in the coupling analysis we need to supplement the extended Higgs sector for example with an additional strongly interacting fermion. In addition, the non-diagonal photon-Z-Higgs is missing, but can be trivially added to this ansatz as we will discuss. We have shown that a SFITTER analysis of the aligned two-Higgs-doublet model - with coupling deviations $\Delta$ computed from the underlying parameters — and the weak scale Higgs coupling analysis indeed give identical results [18]. Furthermore, neglecting the invisible decays and setting $\Delta_{W}=\Delta_{Z}$ the Lagrangian in eq. (2.2) corresponds to the nonlinear effective Lagrangian [63-67] of the Higgs sector, but restricted to the leading terms of the expansion defined in ref. [68].

Our couplings approach only tracks a deviation in the leading coupling of the Higgs boson to each SM particle. We only consider the dimension-4 Lagrangian plus non-decoupling 


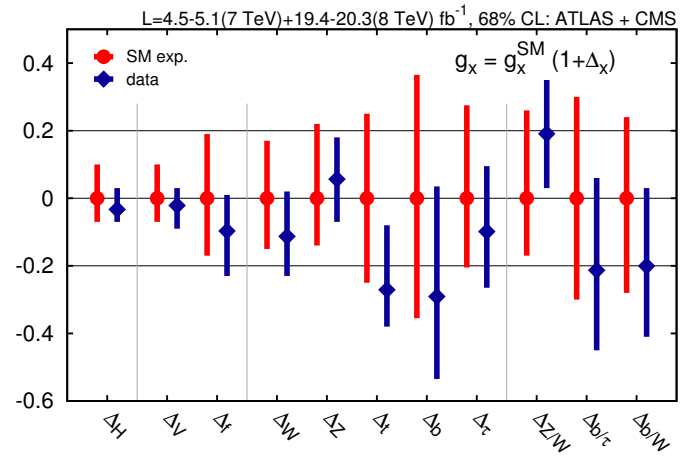

Figure 1. $68 \%$ CL error bars on the deviations $\Delta_{x}$ from all Standard Model couplings of the observed Higgs boson. In this fit we do not allow for new particles in the effective Higgs couplings to photons and gluons, $\Delta_{\gamma}=0=\Delta_{g}$. The results labelled 'SM exp' assume central values on the Standard Model expectation, but the current data error bars.

dimension-6 operators coupling the Higgs to photons or gluons. Strictly speaking, in the linear effective Lagrangian approach this kind of dimension- 6 operators also modify the Higgs couplings to electroweak gauge bosons. As long as the experimental analysis is limited to total rates, we can expect loop-induced corrections for the $H W W$ coupling to be suppressed with respect to shifts in the tree-level coupling $g_{W}$.

A shortcoming of the approach is that it does not include effects of the additional new particles on the SM Lagrangian outside the Higgs sector. For example, it does not link deviations in Higgs couplings to anomalous triple gauge couplings. For a comprehensive analysis of all effects of such new states we have to extend the free Higgs couplings to an effective field theory based on a non-linear sigma model in the broken phase of the electroweak symmetry, see for instance ref. [63-67].

Going beyond the original SFITTER ansatz indeed means re-writing and extending the Lagrangian by additional operators which couple Standard Model fields to the Higgs boson. These operators can be classified by their dimension. For example for the Higgs coupling to $W$ and $Z$ bosons this question has been studied independently of the coupling strength [19-30, 63-67].

\subsection{Standard Model couplings}

In the first step we can fit the five tree level Higgs couplings to all Standard Model particles relevant for the LHC observations. The result is shown in figure 1. The red bars labelled 'SM exp' show results where we have injected a Standard Model Higgs signal on top of the background, i.e. the measured rate in each channel is exactly the SM expectation, but leave everything else unchanged. They indicate that the observed errors are slightly smaller than expected. This is a universal effect of the theoretical uncertainties which we will discuss in detail in section 2.3.

The simplest model, motivated for example by a Higgs portal [69-75] or a single form factor from a strongly interacting Higgs sector [77], consists of a universal coupling modification $\Delta_{H}$. Such a coupling modification is constrained to around $3 \%$ at $68 \% \mathrm{CL}$, in 

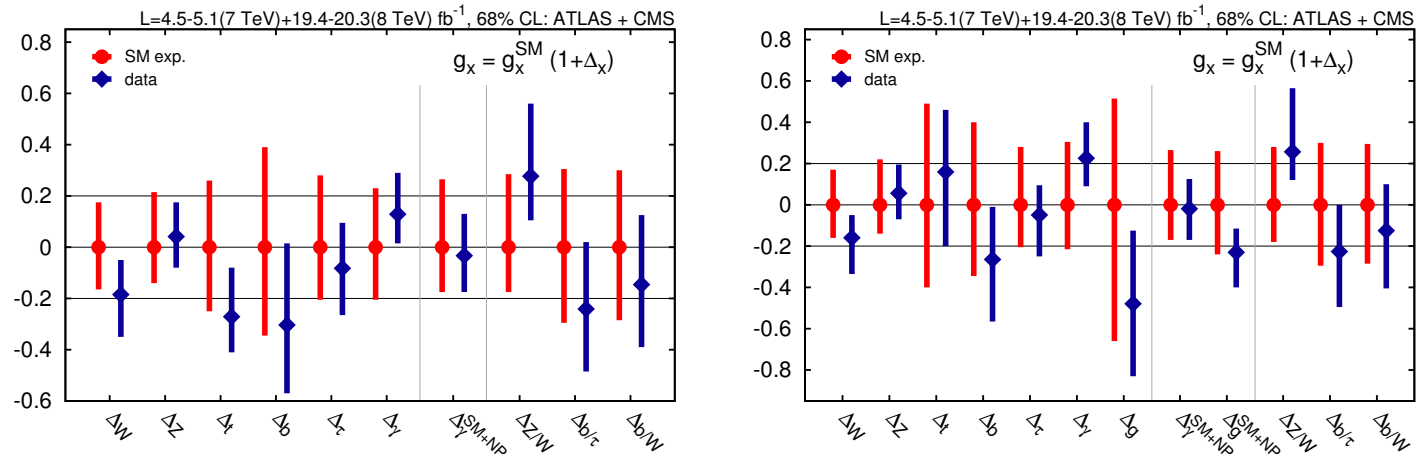

Figure 2. $68 \%$ CL error bars on the deviations $\Delta_{x}$ from all Standard Model couplings of the observed Higgs boson. For the loop-induced couplings we allow new contributions to the $H \gamma \gamma$ coupling only $\left(\Delta_{g}=0\right.$, left) and to the $H \gamma \gamma$ and $H g g$ couplings (right). The results labelled 'SM exp' assume central values on the Standard Model expectation, but the current data error bars.

agreement with the Standard Model or $\Delta_{H}=0$. Translated into a mixing angle $\alpha$ from the Higgs portal whose preferred range at $68 \%$ CL is

$$
\cos \alpha=1+\Delta_{H} \in[0.93,1.03] .
$$

The second simplest model is a universal coupling modification for the Higgs interaction with the gauge bosons $\Delta_{V}$, and one modification for the coupling with the fermions $\Delta_{f}$. Possible ultraviolet completions are given by models with additional Higgs multiplets beyond singlets or doublets, where certain combinations allow us to circumvent the otherwise strong limits by electroweak precision data $[78,79]$. In this case, $\Delta_{V}$ is still constrained to around $\pm 6 \%$ at $68 \%$ CL, while the fermionic coupling shows a reduced precision of around $\pm 12 \%$ at $68 \%$ CL, all consistent with the Standard Model.

An independent variation of five Higgs couplings is also in complete agreement with the Standard Model. Again, the actual error bars on the $\Delta_{x}$ are slightly smaller than what we would expect from exact Standard Model values, an effect we will discuss in section 2.3. The $15 \%$ measurement of the top Yukawa coupling is driven by the Higgs couplings to photons and gluons under the assumption that no new particles contribute to these loop-induced couplings. The measurement of the bottom Yukawa benefits from the normalization of all rates, because in the Standard Model the total width is largely driven by the partial decay width $H \rightarrow b \bar{b}$.

In addition to the individual couplings we also show the deviations of ratios of couplings. Such ratios are useful to remove systematic and theoretical uncertainties. Indeed, we see that the ratio

$$
\frac{g_{b}}{g_{W}}=\frac{g_{b}^{\mathrm{SM}}}{g_{W}^{\mathrm{SM}}}\left(1+\Delta_{b / W}\right)
$$

shows a smaller variation than $\Delta_{b}$ alone. The corresponding positive correlation of $\Delta_{b}$ and $\Delta_{W}$ arises from the total width in the denominator of the predicted event numbers.

Because the Higgs decay $H \rightarrow \gamma \gamma$ has been precisely measured at the LHC we can extend the coupling fit by a new physics contribution to this loop-induced coupling. Of 

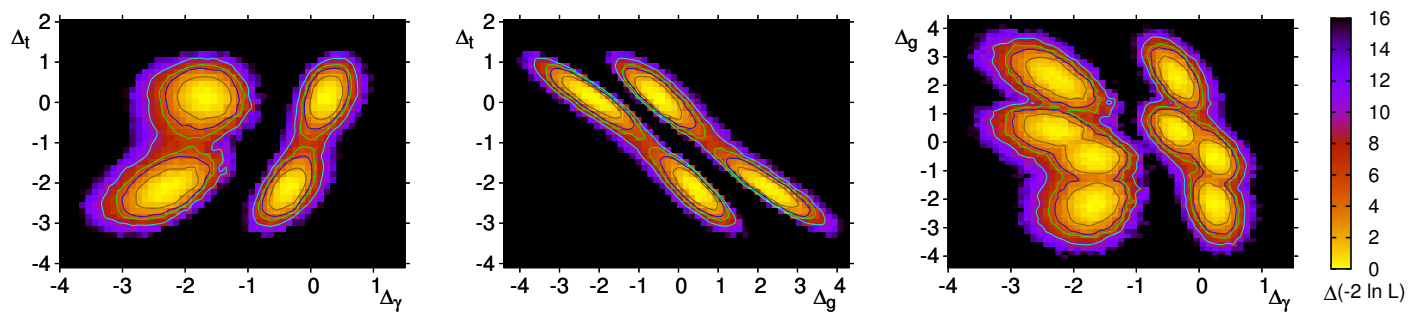

Figure 3. Correlations between different coupling modifications for the fit including $\Delta_{\gamma}$ as well as $\Delta_{g}$. The 1-dimensional profile likelihoods correspond to the results shown as the blue bars in the right panel of figure 2 .

course, the variations of the Standard Model couplings $\Delta_{b, t}$ and $\Delta_{W}$ are consistently reflected in the full $\Delta_{\gamma}^{\mathrm{SM}+\mathrm{NP}}$. Following eq. (2.1) this deviation consists of two terms, the parametric shift from the Standard Model loops and an additional shift from new physics. The latter is shown as part of the coupling measurement in the left panel of figure 2, where we present the results of the six parameter analysis.

The only modification with respect to the fit with $\Delta_{\gamma}=0$ is a slight downward shift of the central value of $\Delta_{W}$. It decreases the contribution from the dominant $W$-loop and therefore has to be compensated by a small positive new physics contribution $\Delta_{\gamma} \sim 0.13$. This also leads to a very slight increase in the uncertainty on $\Delta_{W}$ and $\Delta_{t}$. While the new physics contribution $\Delta_{\gamma}$ based on all available ATLAS and CMS analyses has a one-sigma preference for an additional contribution, the combination $\Delta_{\gamma}^{\mathrm{SM}}+\mathrm{NP}$ is in perfect agreement with the Standard Model. The error bars for $\Delta_{\gamma}$ and $\Delta_{\gamma}^{\mathrm{SM}+\mathrm{NP}}$ have the same size, which means that the interference structure between $\Delta_{W}$ and $\Delta_{t}$ breaks any strong correlation with $\Delta_{\gamma}$ in this fit.

Finally, we show in the right panel of figure 2 the first SFitTer Higgs couplings fit including a new physics contribution to the effective Higgs-gluon coupling, $\Delta_{g} \neq 0$. As argued in section 2 and suggested by our notation, in this seven parameter analysis we focus on the SM-like solutions, i.e. small values of the $\left|\Delta_{x}\right|$. As expected, the increase in the error bar of $\Delta_{t}$ is dramatic. The central value of $\Delta_{t}$ increases by one standard deviation of the new measurement, while $\Delta_{g}$ resides about one standard deviation below the Standard Model expectation, keeping the Higgs production rate close to the Standard Model prediction. Larger deviations of $\Delta_{t}$ are forbidden by constraints on the $t \bar{t} H$ channels. For example, the combined CMS analysis of $t \bar{t} H$ channels reports a signal strength of $2.8_{-0.9}^{+1.0}$ [58], and a specific CMS analysis of the $t \bar{t} H \rightarrow t \bar{t} b \bar{b}$ channel arrives at a signal strength of $1.2_{-1.5}^{+1.6}$ [59]. Typical uncertainties around $100 \%$ on the cross section translate into a $30 \%$ uncertainty on the top Yukawa coupling.

One reason to consider the $t \bar{t} H$ measurements with care is that their significance hardly adds to an independent evidence for this production channel. For example, the combination of ref. [58] rules out the Standard Model at two standard deviations, just slightly less significantly than it establishes the $t \bar{t} H$ production process. An appropriate hypothetical question to ask is if these results would have been published the same way if the signature had been a sign of physics beyond the Standard Model instead of a very much expected signal. 
Aside from the large error bars these measurements in the $t \bar{t} H$ channel have less obvious control of the signal kinematics than other Higgs channels; for example, they might or might not include a clear Higgs mass reconstruction, which is crucial for the unambiguous interpretation of the rate measurement but poses a well known combinatorics problem [8086]. Such a global analysis does lead to a valid upper limit on the $t \bar{t} H$ cross section, but for a lower limit we need to assume that $t \bar{t} H$ production is the only source of relevant events.

As expected, the individual error bars for $\Delta_{g}$ and $\Delta_{t}$ are around three times as large as the error bar for the combination $\Delta_{g}^{\mathrm{SM}+\mathrm{NP}}$, where the latter is known to better than $20 \%$. The remaining Higgs couplings are again hardly affected by the additional parameter $\Delta_{g}$. The error bar of $\Delta_{\gamma}$ is slightly increased because of the enlarged error bar on $\Delta_{t}$. Unlike for $\Delta_{\gamma}$ this is a signal for a very strong correlation between $\Delta_{t}$ and $\Delta_{g}$ in the 2-dimensional profile likelihood.

In figure 3 we show some relevant 2-dimensional correlations of coupling modifications as obtained for the discussed analysis spanning the seven coupling modifications. First, we see that in the $\Delta_{t}$ vs $\Delta_{\gamma}$ plane there are four solutions corresponding to a sign flip in each of the two couplings. We fix the global sign of all Higgs couplings to $\Delta_{W}>-1$ [10]. As long as we limit our analysis to total rates each individual coupling modification at tree level will show a perfect degeneracy between $\Delta_{x}=0$ and $\Delta_{x}=-2$. The loop-induced Higgs-gluon coupling is dominated by the top loop, with a small contribution from the bottom quark, so it will not lift this degeneracy. In contrast, the Higgs-photon coupling is strongly sensitive to the relative sign of the top and $W$-contributions.

The moderate positive correlation in the SM-like solution reflects the fact that an increase of the top Yukawa coupling leads to a decrease in the $H \gamma \gamma$ coupling and hence has to be compensated by a positive value of $\Delta_{\gamma}$. As shown in the central panel the correlation between $\Delta_{t}$ and $\Delta_{g}$ is the strongest correlation in the Higgs couplings analysis. It reflects the fact that the gluon fusion Higgs cross section constrains the sum of the two with a slight re-weighting from the top mass dependence of the loop-induced Higgs-gluon coupling [87]. We will come back to this aspect when discussing the effective theory analysis in section 3 and top mass effects in section 4 . The resulting correlation of $\Delta_{\gamma}$ and $\Delta_{g}$ first of all features eight solutions, arising from the indirect combination through $\Delta_{t} \sim-2,0$. They are clearly separated into the two regimes $\Delta_{\gamma}=-2,0$, while in $\Delta_{g}$ they are merged through the strong correlation with $\Delta_{t}$. For example in the SM-like regime the correlation between the two loop-induced couplings is at a similarly weak level as the correlation between $\Delta_{\gamma}$ and $\Delta_{t}$.

Without showing any detailed results we can also take advantage of the first studies of the Higgs interaction with a photon and a $Z$ boson $[49,50]$. We include a new physics contribution to the loop-induced vertex in the Standard Model, in complete analogy to the modifications $\Delta_{\gamma}$ and $\Delta_{g}$ in eqs. (2.1) and (2.2). The corresponding 68\% CL allowed region on $\Delta_{Z \gamma}$ bounds $\Delta_{Z \gamma}<0.7$ (1.8 at $95 \%$ CL), without any visible effect on the rest of studied parameters shown in figure 2 .

\subsection{Invisible decays}

Higgs decays to invisible particles can only be observed in Higgs production channels with a measurable recoil system. Examples are weak boson fusion [88, 89] and $Z H$ production [90], 


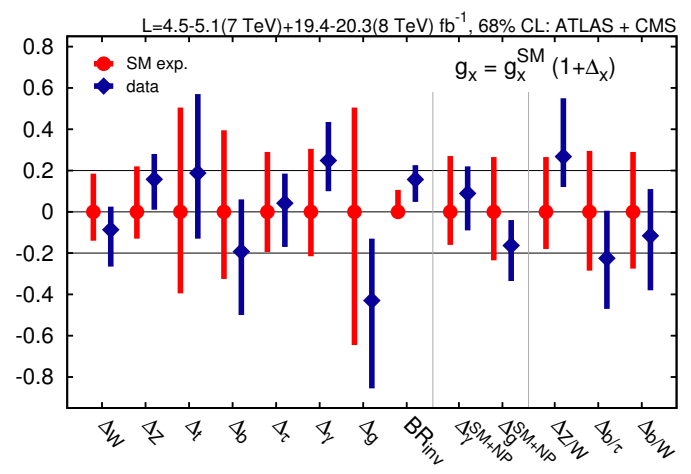

Figure 4. $68 \%$ CL error bars on the deviations $\Delta_{x}$ from all Standard Model couplings of the observed Higgs boson. In addition to all couplings predicted by the Standard Model we include a Higgs decay to invisible particles. The results labelled 'SM exp' assume central values on the Standard Model expectation, but the current data error bars.

where the more sensitive weak boson fusion (WBF) channel might be able to probe invisible branching ratios to $2-3 \%$ with an ultimate integrated luminosity of $3000 \mathrm{fb}^{-1}[88,89]$. To date there are ATLAS and CMS analyses available in these two channels [51-56].

In figure 4 we show the status for the full set of SM Higgs couplings and a hypothetical Higgs coupling to invisible states. Unlike for the other couplings we do not define a coupling deviation $\Delta_{\text {inv }}$, but directly refer to the invisible branching ratio $\mathrm{BR}_{\text {inv }}$. In the Standard Model this invisible branching ratio is generated by the decay $H \rightarrow Z Z^{*} \rightarrow 4 \nu$. It only reaches around $1 \%$ and is therefore unlikely to ever be observed at the LHC. The current limit on invisible Higgs decays in the full Higgs couplings analysis is around $10 \%$. Obviously, for a dedicated analysis with a more constraining model assumption the limits will be stronger [14-17].

Both relevant production processes responsible for invisible Higgs decay searches are mediated by the $Z Z H$ and $W W H$ interactions, where $\Delta_{W, Z}$ are the best measured couplings in the analysis described in section 2.1. The additional searches for invisible decays will not add any new information on the determination on $\Delta_{W, Z}$, so we expect the invisible branching ratio to be orthogonal to the other Higgs coupling measurements, i.e. uncorrelated with all other channels. A slight correlation of the invisible contribution to the total Higgs width leads to a minor upwards shift of all other couplings.

In figure 5 we show the 2-dimensional profile likelihoods for $\mathrm{BR}_{\text {inv }}$ versus the $\Delta_{W}$ and $\Delta_{Z}$ appearing in the production processes and with $\Delta_{b}$ dominating the total width. None of them show a significant correlation. In the absence of strong correlations with any other model parameters in the Lagrangian of eq. (2.2), our best fit value and 68\% CL limits on an invisible Higgs branching ratio $\mathrm{BR}_{\text {inv }}=0.16_{-0.11}^{+0.07}$ will hardly depend on the assumptions for example made about the loop-induced Higgs couplings. The mild preference for a positive invisible branching ratio is driven by the combination of the several experimental searches for an invisible Higgs decay [51-56], as well as the rest of data points considered in the analysis, as listed in section 1.1. Specially relevant is the deficit of events measured in the ATLAS $V H, H \rightarrow b \bar{b}$ search [47], that can be better accommodated by a negative central 

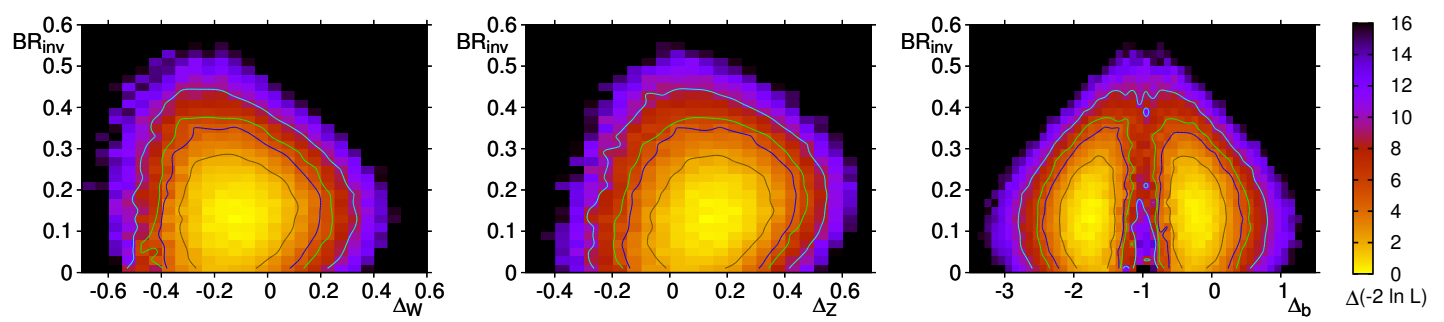

Figure 5. Correlations between different coupling modifications to SM particles and the invisible branching ratio. The corresponding 1-dimensional profile likelihoods are shown as the blue bars in figure 4.

value for $\Delta_{b}$, together with a small positive value for $\mathrm{BR}_{\mathrm{inv}}$ such that the rest of decay channels are barely affected.

\subsection{Theoretical uncertainties}

In this last part of the Higgs couplings analysis we highlight open questions related to the treatment of theoretical uncertainties. Unlike experimental uncertainties, the estimate of for example higher-order contributions missing in the calculation of an LHC cross section do not offer a frequentist interpretation. It is unclear what kind of likelihood distribution of the associated nuisance parameters we need to assume. On the other hand, once we define a likelihood distribution for these nuisance parameters the rest of the likelihood analysis is completely defined. ${ }^{2}$

All we can say from a theory perspective is that a certain deviation from the best available cross section or rate prediction is in some kind of agreement with the Standard Model or beyond the level where we are willing to consider such an interpretation. This problem is independent of the way we determine the uncertainty range on an observable. Varying the unphysical factorization and renormalization scales is only one method, and there might be many others. If we assume a flat distribution for the theoretical uncertainty to remove any bias between different predictions within the allowed range, the RFIT scheme [91] is uniquely defined as the profile likelihood combination with the experimental uncertainties. Uncertainties on the parton densities are treated in complete analogy to the theoretical uncertainties from unknown higher orders, including any assumption on their correlations.

Note that a similar problem of choosing a prior for the theoretical uncertainty arises in the Bayesian approach [92, 93]. On the one hand the Bayesian approach allows for a choice of priors in general, including the theoretical uncertainty, without having to ask for a statistical interpretation. On the other hand, this renders one assumption on the prior as ad-hoc as any other. This leaves us with the crucial task to carefully check the prior dependence of our result.

No matter what approach we follow, it is important to recognize that we should employ a conservative estimate of theoretical uncertainties in addition to a flexible framework

\footnotetext{
${ }^{2}$ Because of this lack of uniqueness in the definition of theoretical uncertainties we advocate for not including them in the experimental analyses or (if unavoidable) for factoring them out to allow for a flexible analysis [76].
} 

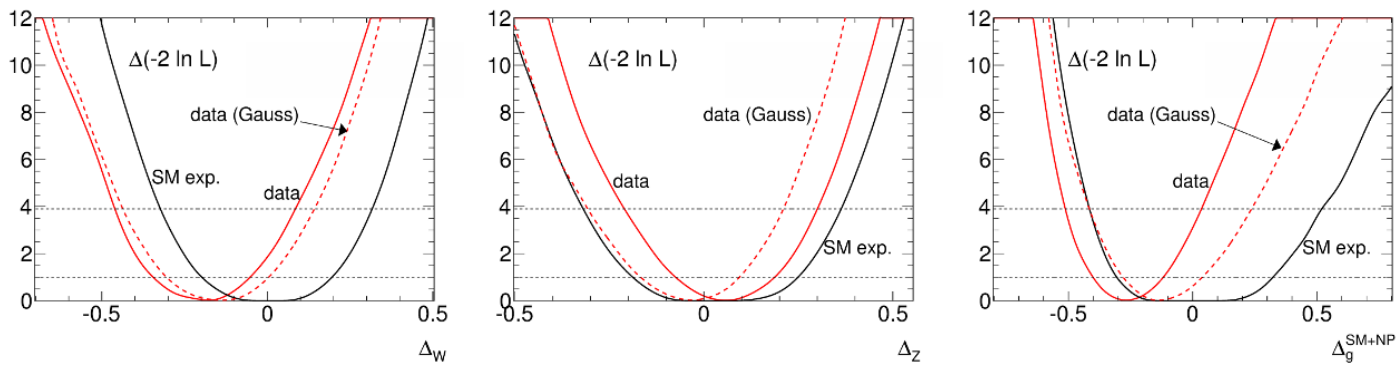

Figure 6. 1-dimensional profile likelihoods for $\Delta_{W}, \Delta_{Z}$, and $\Delta_{g}^{\mathrm{SM}+\mathrm{NP}}$. We assume all measurements on the Standard Model values with flat theoretical uncertainties (black), observed rates with flat (solid red) and Gaussian (dashed red) theoretical uncertainties.

which allows us to test different assumptions as efficiently as possible [76]. This includes the shape of the likelihood associated with the theoretical error bars as well as the size of the theoretical error bars. Before we discuss the modelling of the likelihood including a theoretical uncertainty we need to understand an effect which we observe all through section 2.1 and 2.2: the expected size of the error bars is consistently larger than the observed errors. The reason is linked to the behavior of flat theoretical uncertainties once the measurements start developing a pull. In figure 6 we show a set of 1-dimensional profile likelihoods. For the expected limits, i.e. assuming that all rate measurements agree perfectly with the Standard Model, we clearly see the flat central range, induced by the theoretical uncertainties.

However, once we allow for a statistical distribution of the measurements all 1dimensional profile likelihoods lose the flat central regions and instead follow the Gaussian shape of the dominant experimental uncertainties. Note that this does not have to be the case based on first principles: if all uncertainties were flat in the rates, the resulting profile likelihood would keep its box shape, and the errors would be added linearly [91, 94]. The central limit theorem does not guarantee a Gaussian distribution, because the profile likelihood does not involve a convolution. The resulting Gaussians in figure 6 instead reflect the fact that theoretical uncertainties are smaller than their experimental counter parts, and the Gaussian features of the latter dominate the final distribution once we allow for a spread of measurements. The curves in figure 6 illustrate the general observation, that actual 1-dimensional error bands with their Gaussian behavior are smaller than the expected errors with their flat central range, once we include real data.

With this observation in mind we show a set of results from our systematic study of the appropriate treatment of theoretical uncertainties in figure 7. The SFITTER standard approach to theoretical uncertainties is based on

- uncorrelated uncertainties for the production, to account for very different kinematic selections;

- an allowed cross section range given by the scale dependence of the best available prediction; 

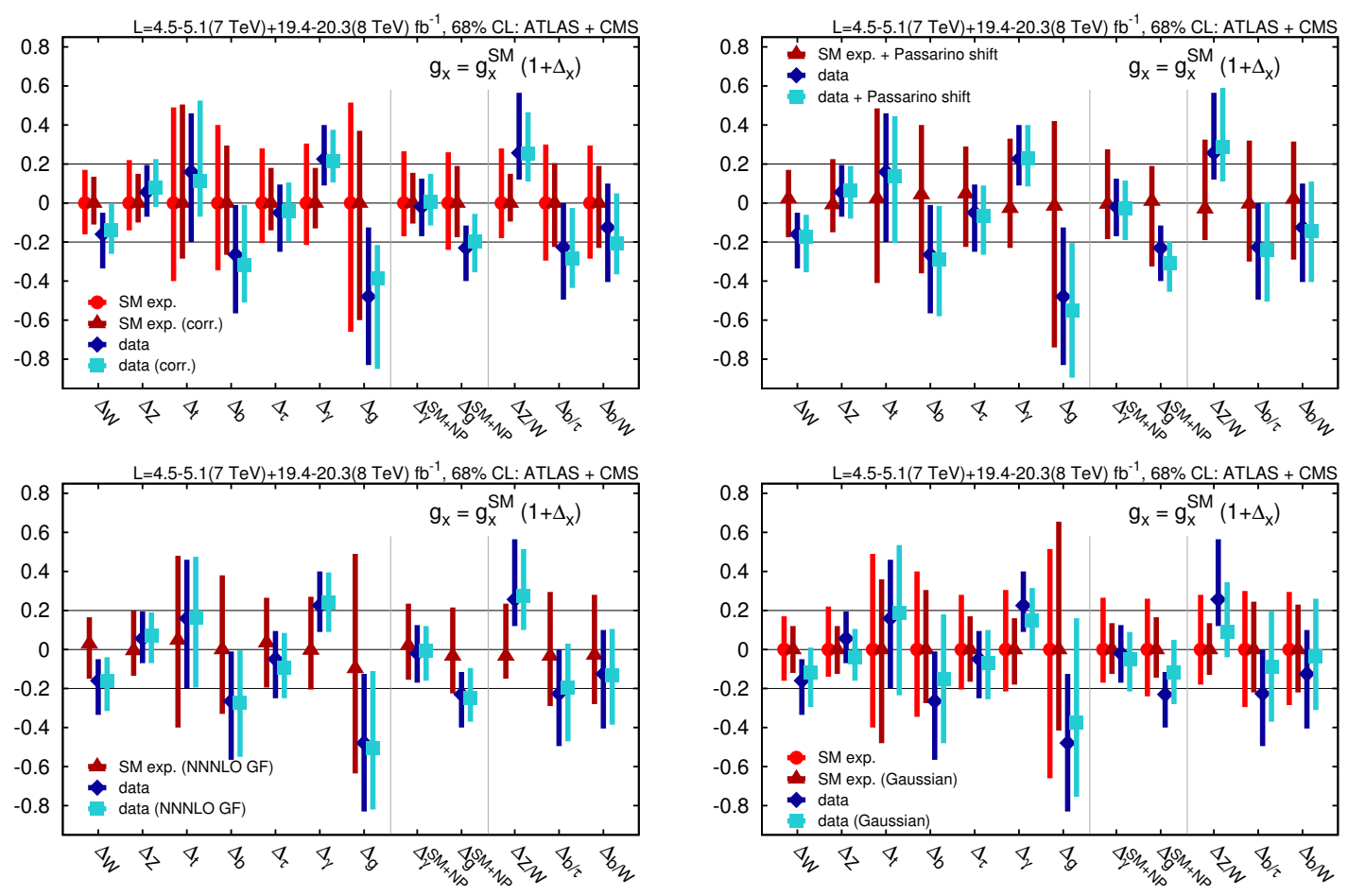

Figure 7. $68 \%$ CL error bars on the deviations $\Delta_{x}$ from all Standard Model couplings of the observed Higgs boson. First, we show the effect of fully correlated theoretical uncertainties on the different production processes (upper left); next, we show the results with the modified theoretical uncertainties proposed in ref. [95] (upper right); then, we illustrate the effect of the $\mathrm{N}^{3} \mathrm{LO}$ calculation of the Higgs cross section [96] (lower left); finally, we illustrate what happens when we simulate the theoretical uncertainties with a Gaussian distribution (lower right). The results labelled 'SM exp' assume central values on the Standard Model expectation, but the current data error bars.

- a flat likelihood distribution of the associated nuisance parameter for the cross sections and the decays.

These three assumptions we check one-by-one. In the upper left panel of figure 7 we show an alternative SFITTER analysis with fully correlated uncertainties for the production rates, including the error bar from the parton densities. Because of the strong correlations between the different production and decay processes expressed in terms of Higgs couplings such a shift in the assumed correlations for the theoretical uncertainties could have a significant effect. However, we see that for full correlations the size of the error bars is only slightly reduced, in spite of the fact that the central values for example for $\Delta_{b}$ shifts by half a standard deviation. The truth lies somewhere in between the fully correlated and the fully uncorrelated theoretical uncertainties, where for the upcoming Run II there will be a tendency towards less correlation because of the more specific analysis strategies. On the other hand, the difference between fully uncorrelated and fully correlated errors is not worrisome and we stay on the conservative, uncorrelated side.

In the upper right panel of figure 7 we show the change in the extracted Higgs couplings when we modify the assumed theoretical uncertainties following ref. [95]. For a collider 
energy of $8 \mathrm{TeV}$ and a Higgs mass of $125 \mathrm{GeV}$ the default prediction of the gluon fusion production cross section is

$$
\sigma_{p p \rightarrow H}=19.52 \mathrm{pb} \pm 7.5 \%_{\text {pert }} \pm 7.2 \%_{\mathrm{pdf}} .
$$

Using the modified definition of ref. [95] it becomes

$$
\sigma_{p p \rightarrow H}=22.49 \mathrm{pb} \pm 13 \%_{\text {pert }} \pm 7.2 \%_{\text {pdf }} .
$$

The change in central values as well as in the size of the error bars is hardly observable, in spite of the sizeable change in the size of the theoretical uncertainties. This confirms the earlier observation that theoretical uncertainties are subleading for the Run I analysis.

Also related to the theoretical uncertainty we include the recent computation of the $\mathrm{N}^{3} \mathrm{LO}$ corrections to the Higgs production rate at the LHC [96]. The corresponding results are shown in the lower left panel of figure 7 . Now the cross section prediction reads

$$
\sigma_{p p \rightarrow H}=19.95 \mathrm{pb} \pm 1.5 \%_{\text {pert }} \pm 7.2 \%_{\mathrm{pdf}}
$$

with a significantly more optimistic error based on the central scale choice $\mu_{R, F}=m_{H} / 2$. Again, the now strongly reduced theoretical uncertainty hardly affects the Run I results.

Finally, we compare the precision of the Higgs couplings determination with flat theoretical uncertainties with a Gaussian nuisance parameter. The main differences between the frequentist RFIT treatment and Gaussian theoretical uncertainties are not related to the shape of the final distribution, but to the size of the combined theoretical uncertainties. First, combining two flat theoretical uncertainties, for example from unknown higher orders and the parton densities, will lead to a linear combination of the two error bars in the frequentist RFIT scheme [91, 94, 97-100]. In the Gaussian approach they are added in quadrature. Second, it is not clear with which Gaussian significance we should identify the ends of the box-shaped distribution. For example, computing the standard deviation of a flat data set stays well below the size of the box. This means that if we compare the range of one standard deviation for the RFIT scheme with one standard deviation of the Gaussian, the error on the flat distribution appears smaller.

Per se, it is not clear which of the two effects will dominate in a given fit. In this situation we could choose the flat and Gaussian theoretical uncertainties without a clear preference. We stick to the former because we assume that it will be the conservative approach once theoretical errors actually affect LHC results with larger data sets.

\section{Higgs operators}

Going beyond a measurement of all couplings predicted by the Standard Model we can ask a different question: Which consistent Lagrangian describes all LHC measurements best? A standard approach is defined by effective field theory [101-103], where we categorize a Lagrangian with the appropriate symmetries in terms of the expansion parameter. While the results of the previous section can be interpreted in the framework of a non-linear effective Lagrangian approach as we have explained, in this section we focus on the linear 
case. In the linear sigma model we construct a $\mathrm{SU}(2)_{L} \times \mathrm{U}(1)_{Y}$-symmetric Higgs Lagrangian based on the doublet $\Phi$ and order it according to the inverse powers of the cutoff scale, $1 / \Lambda[78,79,104-113]$. The Lagrangian, here restricting to all dimension- 6 operators

$$
\mathcal{L}=\sum_{x} \frac{f_{x}}{\Lambda^{2}} \mathcal{O}_{x}
$$

is gauge invariant, but not fully renormalizable or unitary.

Strictly speaking, in the SM Higgs sector we should separate two sources of dimension6 operators. Yukawa couplings or gauge boson couplings from spontaneous symmetry breaking violate the Appelquist-Carazzone decoupling theorem [114], which means that the Higgs couplings to photons and gluons are only suppressed by $1 / v$. New physics generally gives rise to dimension- 6 operators suppressed by $1 / \Lambda^{2}$, leading to Higgs coupling strengths to photons and gluons scaling like $v / \Lambda^{2}$. This distinction will be reflected in the normalization of the respective operators below.

\subsection{Dimension-6 operator basis}

Before we present the result of the LHC analysis we need to define our basis of dimension- 6 operators. The minimum independent set of dimension-6 operators with the SM particle content (including the Higgs boson as an $\mathrm{SU}(2)_{L}$ doublet) and compatible with the $\mathrm{SM}$ gauge symmetries as well as baryon number conservation contains 59 operators, up to flavor and Hermitian conjugation [113]. To present our choice of operator basis [19-21], we start by imposing $C$ and $P$ invariance and employing for the bosonic sector the classical non-minimal set of dimension-6 operators in the HISZ basis [111, 112], with the following operators contributing to the Higgs interactions with gauge bosons:

$$
\begin{aligned}
& \mathcal{O}_{G G}=\Phi^{\dagger} \Phi G_{\mu \nu}^{a} G^{a \mu \nu} \\
& \mathcal{O}_{W W}=\Phi^{\dagger} \hat{W}_{\mu \nu} \hat{W}^{\mu \nu} \Phi \\
& \mathcal{O}_{B B}=\Phi^{\dagger} \hat{B}_{\mu \nu} \hat{B}^{\mu \nu} \Phi \\
& \mathcal{O}_{B W}=\Phi^{\dagger} \hat{B}_{\mu \nu} \hat{W}^{\mu \nu} \Phi \\
& \mathcal{O}_{W}=\left(D_{\mu} \Phi\right)^{\dagger} \hat{W}^{\mu \nu}\left(D_{\nu} \Phi\right) \\
& \mathcal{O}_{B}=\left(D_{\mu} \Phi\right)^{\dagger} \hat{B}^{\mu \nu}\left(D_{\nu} \Phi\right) \\
& \mathcal{O}_{\Phi, 1}=\left(D_{\mu} \Phi\right)^{\dagger} \Phi \Phi^{\dagger}\left(D^{\mu} \Phi\right) \\
& \mathcal{O}_{\Phi, 2}=\frac{1}{2} \partial^{\mu}\left(\Phi^{\dagger} \Phi\right) \partial_{\mu}\left(\Phi^{\dagger} \Phi\right) \\
& \mathcal{O}_{\Phi, 4}=\left(D_{\mu} \Phi\right)^{\dagger}\left(D^{\mu} \Phi\right)\left(\Phi^{\dagger} \Phi\right) \text {. }
\end{aligned}
$$

Here the Higgs doublet covariant derivative is $D_{\mu} \Phi=\left(\partial_{\mu}+i g^{\prime} B_{\mu} / 2+i g \sigma_{a} W_{\mu}^{a} / 2\right) \Phi$, the hatted field strengths are $\hat{B}_{\mu \nu}=i g^{\prime} B_{\mu \nu} / 2$ and $\hat{W}_{\mu \nu}=i g \sigma^{a} W_{\mu \nu}^{a} / 2$, with the Pauli matrices written as $\sigma^{a}$. The $\mathrm{SU}(2)_{L}$ and $\mathrm{U}(1)_{Y}$ gauge couplings are $g$ and $g^{\prime}$. The additional operator $\mathcal{O}_{\Phi, 3}=\left(\Phi^{\dagger} \Phi\right)^{3}$ is crucial for the structure of the Higgs potential and for a theoretical interpretation of the measurement of the Higgs self-coupling, but we can safely omit it for the LHC Run I analysis.

The final choice of structures for our global Higgs analysis follows ref. [19-21], relying on operators contributing to existing data. We first use the equations of motion (including all necessary fermionic operators [113] omitted in this brief introduction) to rotate to a basis where there are not blind directions linked to electroweak precision data. We then neglect all operators contributing to the bulk of electroweak precision data at tree level; their coefficients will be too constrained to lead to observable deviations in LHC Higgs measurements. After using the remaining equation of motion to remove redundancy, we finally neglect all operators that we know will not be constrained by LHC Higgs measurements. 
We are left with the final set of nine operators that parametrize the Higgs interactions at the LHC. For the gauge boson interactions they are

$$
\mathcal{L}_{\text {eff }}^{H V V}=-\frac{\alpha_{s}}{8 \pi} \frac{f_{G G}}{\Lambda^{2}} \mathcal{O}_{G G}+\frac{f_{B B}}{\Lambda^{2}} \mathcal{O}_{B B}+\frac{f_{W W}}{\Lambda^{2}} \mathcal{O}_{W W}+\frac{f_{B}}{\Lambda^{2}} \mathcal{O}_{B}+\frac{f_{W}}{\Lambda^{2}} \mathcal{O}_{W}+\frac{f_{\Phi, 2}}{\Lambda^{2}} \mathcal{O}_{\Phi, 2} .
$$

The operator $\mathcal{O}_{\Phi, 2}$ appears in the gauge and fermionic Lagrangians, because it leads to a finite renormalization of the Higgs field and hence a universal shift of all Higgs couplings to Standard Model fields [19-21]. This set of dimension-6 effective operators gives rise to the following Higgs interactions with SM gauge boson pairs,

$$
\begin{aligned}
\mathcal{L}^{H V V}= & g_{H g g} H G_{\mu \nu}^{a} G^{a \mu \nu}+g_{H \gamma \gamma} H A_{\mu \nu} A^{\mu \nu}+g_{H Z \gamma}^{(1)} A_{\mu \nu} Z^{\mu} \partial^{\nu} H+g_{H Z \gamma}^{(2)} H A_{\mu \nu} Z^{\mu \nu} \\
& +g_{H Z Z}^{(1)} Z_{\mu \nu} Z^{\mu} \partial^{\nu} H+g_{H Z Z}^{(2)} H Z_{\mu \nu} Z^{\mu \nu}+g_{H Z Z}^{(3)} H Z_{\mu} Z^{\mu} \\
& +g_{H W W}^{(1)}\left(W_{\mu \nu}^{+} W^{-\mu} \partial^{\nu} H+\text { h.c. }\right)+g_{H W W}^{(2)} H W_{\mu \nu}^{+} W^{-\mu \nu}+g_{H W W}^{(3)} H W_{\mu}^{+} W^{-\mu},
\end{aligned}
$$

where $V_{\mu \nu}=\partial_{\mu} V_{\nu}-\partial_{\nu} V_{\mu}$, with $V=A, Z, W, G$. These effective couplings are related to the coefficients in eq. (3.3) through

$$
\begin{aligned}
g_{H g g} & =-\frac{\alpha_{s}}{8 \pi} \frac{f_{G G} v}{\Lambda^{2}} & g_{H Z \gamma}^{(1)} & =\frac{g^{2} v}{2 \Lambda^{2}} \frac{s_{w}\left(f_{W}-f_{B}\right)}{2 c_{w}} \\
g_{H \gamma \gamma} & =-\frac{g^{2} v s_{w}^{2}}{2 \Lambda^{2}} \frac{f_{B B}+f_{W W}}{2} & g_{H Z \gamma}^{(2)} & =\frac{g^{2} v}{2 \Lambda^{2}} \frac{s_{w}\left(2 s_{w}^{2} f_{B B}-2 c_{w}^{2} f_{W W}\right)}{2 c_{w}} \\
g_{H Z Z}^{(1)} & =\frac{g^{2} v}{2 \Lambda^{2}} \frac{c_{w}^{2} f_{W}+s_{w}^{2} f_{B}}{2 c_{w}^{2}} & g_{H W W}^{(1)} & =\frac{g^{2} v}{2 \Lambda^{2}} \frac{f_{W}}{2} \\
g_{H Z Z}^{(2)} & =-\frac{g^{2} v}{2 \Lambda^{2}} \frac{s_{w}^{4} f_{B B}+c_{w}^{4} f_{W W}}{2 c_{w}^{2}} & g_{H W W}^{(2)} & =-\frac{g^{2} v}{2 \Lambda^{2}} f_{W W} \\
g_{H Z Z}^{(3)} & =m_{Z}^{2}\left(\sqrt{2} G_{F}\right)^{1 / 2}\left(1-\frac{v^{2}}{2 \Lambda^{2}} f_{\Phi, 2}\right) & g_{H W W}^{(3)} & =2 m_{W}^{2}\left(\sqrt{2} G_{F}\right)^{1 / 2}\left(1-\frac{v^{2}}{2 \Lambda^{2}} f_{\Phi, 2}\right),
\end{aligned}
$$

where $s_{w}$ and $c_{w}$ stands for the sine and cosine of the weak mixing angle.

We finally focus on the huge set of dimension- 6 operators contributing to the Higgs interactions with fermion pairs [113]. Because of a lack of appropriate observables in the LHC Higgs measurements, from the fermionic operators left in the final basis we limit ourselves to the flavor-diagonal Yukawa structures

$$
\mathcal{O}_{e \Phi, 33}=\left(\Phi^{\dagger} \Phi\right)\left(\bar{L}_{3} \Phi e_{R, 3}\right) \quad \mathcal{O}_{u \Phi, 33}=\left(\Phi^{\dagger} \Phi\right)\left(\bar{Q}_{3} \tilde{\Phi} u_{R, 3}\right) \quad \mathcal{O}_{d \Phi, 33}=\left(\Phi^{\dagger} \Phi\right)\left(\bar{Q}_{3} \Phi d_{R, 3}\right)
$$

with $\tilde{\Phi}=i \sigma_{2} \Phi^{*}$, and where the conventions for the fermion fields are $L$ for the lepton doublet, $Q$ for the quark doublet, and $f_{R}$ for the $\mathrm{SU}(2)_{L}$ singlet fermions. The corresponding effective Lagrangian for the fermionic interactions reads

$$
\mathcal{L}_{\text {eff }}^{H f f}=\frac{f_{\tau} m_{\tau}}{v \Lambda^{2}} \mathcal{O}_{e \Phi, 33}+\frac{f_{b} m_{b}}{v \Lambda^{2}} \mathcal{O}_{d \Phi, 33}+\frac{f_{t} m_{t}}{v \Lambda^{2}} \mathcal{O}_{u \Phi, 33}+\frac{f_{\Phi, 2}}{\Lambda^{2}} \mathcal{O}_{\Phi, 2} .
$$

As mentioned above, $\mathcal{O}_{\Phi, 2}$ affects the Higgs couplings universally. In analogy to the Higgsgluon coupling we scale the fermionic $f_{x}$ by a factor $m / v$ to reflect the chiral nature of the Higgs coupling operator [115-117]. For the Higgs couplings to SM fermions this implies

$$
\mathcal{L}^{H f f}=g_{f} H \bar{f}_{L} f_{R}+\text { h.c. } \quad \text { with } \quad g_{f}=-\frac{m_{f}}{v}\left(1-\frac{v^{2}}{2 \Lambda^{2}} f_{\Phi, 2}-\frac{v^{2}}{\sqrt{2} \Lambda^{2}} f_{f}\right),
$$

where we define the physical masses and fermions in the mass basis $(f=\tau, b, t)$. 

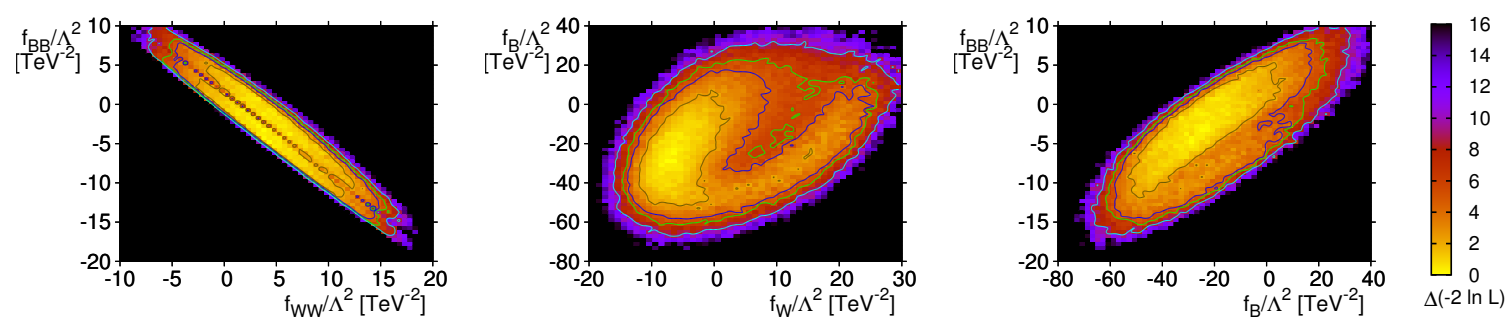

Figure 8. Correlations between different coefficients $f_{x} / \Lambda^{2}$, measured in $\mathrm{TeV}^{-2}$. The 1-dimensional profile likelihoods corresponding to these results are shown as the blue bars in figure 9 .

As we discuss in the following sections, the sensitivity of the current LHC Higgs searches does not allow yet for a model-independent interpretation of the results of an analysis based only on dimension- 6 operators. We thus note that for the results derived in the following we consider the parametrizations in eqs. (3.3)-(3.8) without any further truncation, keeping the quadratic dependence on the dimension-6 operator coefficients.

\subsection{Rate-based analysis}

As a first step we update the global analysis of dimension- 6 operators based on the complete Run I data in the SFiTTER framework. The main difference to the analysis of ref. [19-21] is the variable top-Yukawa operator, which can now be constrained by $t \bar{t} H$ production as well as the Higgs production via gluon fusion.

The contributions of the dimension- 6 operators to the production rates and decay widths are calculated using MadGraph5 [118] and FeynRules [119]. We check our results with CoMPheP [120, 121] and VBFNLO [122, 123]. We approximately include higher-order corrections through $K$-factors computed for the Standard Model processes [97-100]. Similarly, for this rate-based analysis we assume that all detector efficiencies are identical for both the SM Higgs processes and the corresponding dimension- 6 contributions. The results of this 9-parameter global analysis are shown in figure 8 and figure 9, after performing a statistical analysis as described in section 1.2. For the present case we show the multiple degenerate solutions.

In figure 8 we depict a selection of interesting correlations between the dimension- 6 operators. In addition to the correlations discussed in the previous section, e.g. $f_{G G}$ vs $f_{t}$ shown in figure 3 , the dimension- 6 operators introduce a rich structure of correlations related to the Higgs interactions with electroweak gauge bosons. As long as the analysis is only based on rate measurements in the Higgs sector, these correlations are the main difference compared to the $\Delta$-framework. The strongest of these correlations is due to the di-photon channel, as it is measured with the highest precision. Therefore, the tree-level contributions from $f_{W W}$ and $f_{B B}$ to the Higgs coupling to photon pairs generate the strong correlation in the left panel of figure 8; see eq. (3.4). The two, slightly separated, allowed regions at $68 \%$ CL are due to the interference between the dimension- 6 amplitudes and the Standard Model ones. The fact that both $f_{W W}$ and $f_{B B}$ receive their strongest constraints from the di-photon channel, reflects that their contribution in the rate based analysis is very similar to the addition of $\Delta_{\gamma}$ in the previous section. While this strong correlation is 

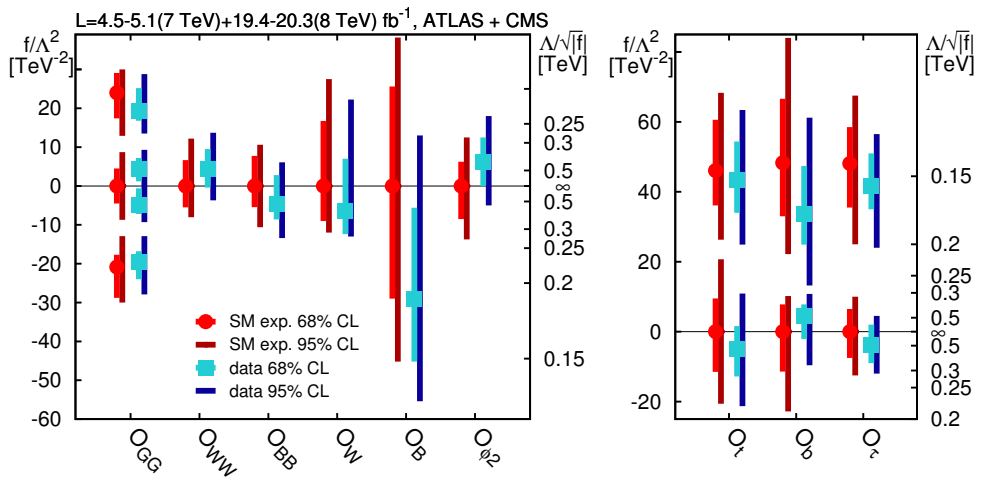

Figure 9. Error bars on the coefficients $f_{x} / \Lambda^{2}$ for the dimension- 6 operators defined in eq. (3.3) (left panel) and eq. (3.7) (right panel). We only include total rate information and show $68 \%$ CL as well as $95 \%$ CL contours. Unlike for the other 1-dimensional profile likelihoods we keep track of the secondary minima in this one figure. The results labelled 'SM exp' assume central values on the Standard Model expectation, but the current data error bars.

partially broken by their smaller contribution to the other channels in the analysis, we will see in the following section that the addition of kinematic distributions will increase the sensitive to $f_{W W}$ and $f_{B B}$ stemming from VBF and Higgs associate production channels.

In the central panel of figure 8 we show the correlation between $f_{B}$ and $f_{W}$. The Wilson coefficient $f_{W}$ is much more strongly constrained than $f_{B}$, because of the large contributions of the former to the $H V V$ vertices $\left(V=Z, W^{ \pm}\right)$while $f_{B}$ only contributes to $H Z Z$ with a weak mixing angle suppression. The mild impact of $f_{B}$ will eventually be compensated by measurements of $H \rightarrow Z \gamma$ decays. Moreover, the contributions to $H V V$ also correlate $f_{B}$ to $f_{W W}$ and $f_{B B}$, as displayed in the right panel of figure 8 .

The universal contribution of $f_{\Phi, 2}$ to all Higgs couplings strongly correlates this operator with the rest of dimension- 6 structures, both in the bosonic and in the fermionic sectors. This way, $f_{\Phi, 2}$ in principle lifts the degeneracy between the two allowed regions for $f_{b}$ and $f_{\tau}$, which is due to the interference between the SM amplitudes and the higherdimensional operators. The actual likelihood values for the two minima are still equivalent though. In the $\Delta$-framework these regions are almost entirely degenerate, allowing us to focus on the SM-like solution in that case.

Starting with the assumption that to first approximation the rate-based analysis of dimension- 6 operators is physically equivalent to the Higgs coupling analysis described in section 2.1 the strong correlations shown in figure 8 still pose a technical problem. The Higgs coupling modifications $\Delta_{x}$ are by definition well aligned with the experimental measurements, which means that the profile likelihood construction down to 1-dimensional likelihoods is straightforward. For example the correlation between $f_{B}$ and $f_{W}$ makes it obvious that a profile likelihood either in $f_{B}$ or $f_{W}$ will have to deal with strongly nonGaussian distributions, including secondary minima.

With this technical caveat in mind we show in figure 9 the best fit points and the corresponding 1-dimensional $68 \%$ and $95 \%$ CL regions for each effective operator. We follow the procedure described in section 1.2, in this case keeping all possible solutions for 
$\mathcal{O}_{t, b, \tau}$ and $\mathcal{O}_{G G}$. As we have discussed the strongest constraints apply to $f_{W W}$ and $f_{B B}$. Next are $f_{W}$ and $f_{\phi, 2}$, and finally the weaker constraint $f_{B}$, as discussed above. Just like for $\Delta_{t}$ the free value of $f_{t}$ enlarges the error bars for $f_{G G}$ and splits the allowed parameter range into more or less distinct regions, like those shown in figures 3 and 9 .

In the right panel we observe the expected secondary solutions for all three $f_{t, b, \tau}$. To compare the errors on the couplings to fermions we should keep in mind that in eq. (3.7) the chiral factor is taken out of the definition of the operator and its associated scale $\Lambda$. As we can see, at the $68 \%$ CL the secondary solutions appear as clear additional structures, while at $95 \%$ CL the SM-like and secondary solutions barely separate for $f_{b}$. This allows us to cleanly separate SM-like solutions from those with merely switched signs of the Yukawa couplings. Note that the latter correspond to a new physics scale $\Lambda \sim 150 \mathrm{GeV}$ in the presence of a chiral symmetry factor, shedding some doubt on the effective theory treatment as a whole.

From a statistical point of view it is not clear how one would deal with such alternative solutions; in our case we show the solutions with flipped signs of the Yukawa couplings in figure 9, but will omit them in the 1-dimensional profile likelihood for the rest of the present section. In the Markov chain analysis they will be of course still included. We will revisit this issue in section 4 for the case of the top Yukawa coupling.

\subsection{Kinematic distributions}

Based exclusively on total event rates, the results from the previous section do not take full advantage of the available information. In eqs. (3.4) and (3.8) we observe that $\mathcal{O}_{\Phi, 2}$, $\mathcal{O}_{b}, \mathcal{O}_{\tau}$, and $\mathcal{O}_{t}$ merely modify the SM coupling strengths, but the other dimension-6 operators do generate new Lorentz structures. These anomalous Lorentz structures are best visible in Higgs production rather than decays, because the momentum flow is not limited by the Higgs mass. Their study is indeed one of the most interesting aspects of our effective field theory analysis.

To establish a framework for an implementation of kinematic distributions into the Higgs operator analysis we first focus on $V H$ production and weak boson fusion. Adding kinematics to our global analysis faces a considerable challenge, because we are limited to fully documented distributions. When multi-variate analysis techniques are applied, the documented distributions are usually not optimized. However for two test cases we will show how we can consistently combine information from rates with kinematic distributions without weakening the analysis.

Finally, the effective Higgs Lagrangian does not define a UV-complete theory if we only include dimension- 6 operators. The cutoff scale $\Lambda$ is encoded in the ansatz, and at least for a weakly interacting theory the experimental sensitivity offers a consistency test. There exist several ways to define a model which we can consistently compare to data:

1. take the alternative model at face value, unless a prediction actually violates unitarity. This approach maximizes the distinguishing power of the measurement, but it only rules out the ultraviolet completion with the least SM-like behavior. 

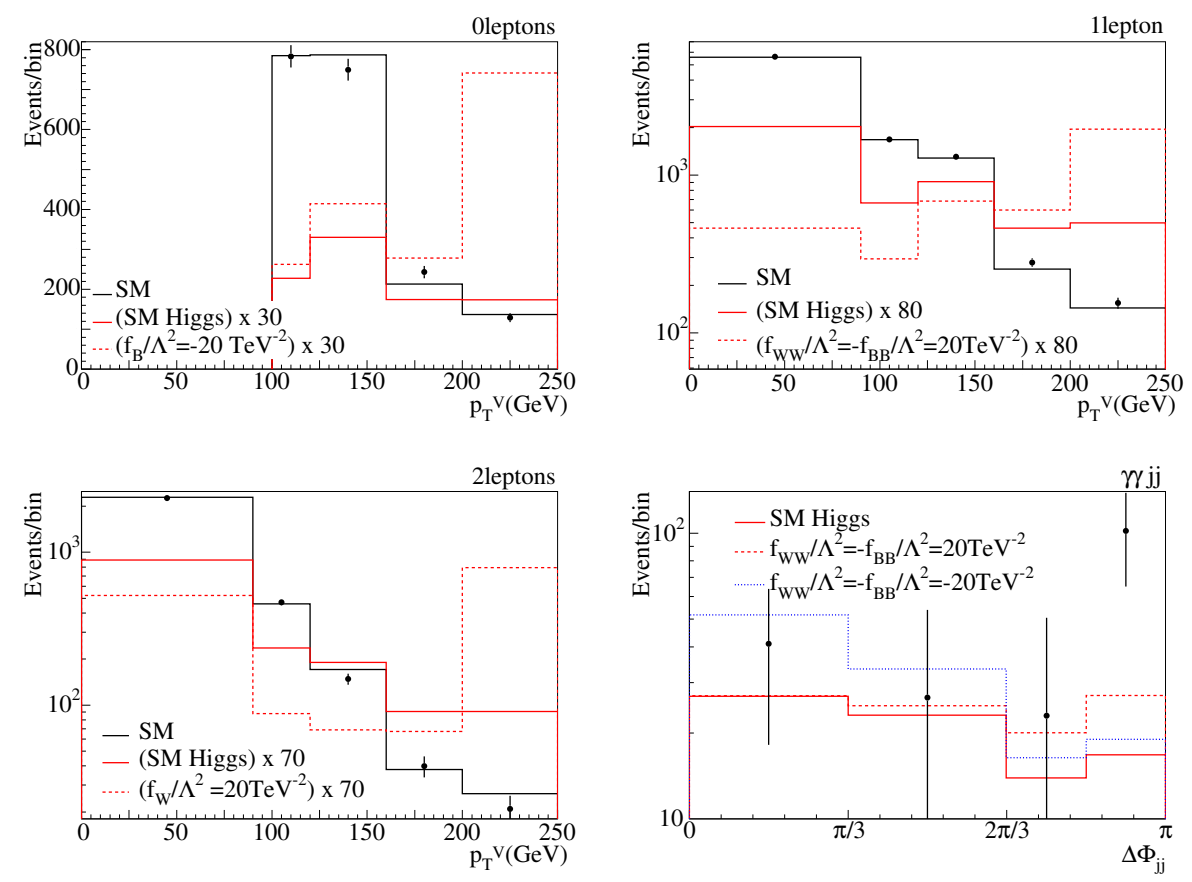

Figure 10. Upper and left lower panels: $p_{T}^{V}$ distributions from $V H$ production [47]. We show the SM Higgs-plus-background expectation (black solid), the number of observed events, the SM Higgs hypothesis (red solid), and the expectation from one dimension-6 operator (red dashed). Lower right: $\Delta \phi_{j j}$ distribution in ref. [60]. We display the number of observed events, the SMHiggs hypothesis (red), and the expectation from adding a set of dimension- 6 operators (dashed red and dotted blue). All plots include $20.3 \mathrm{fb}^{-1}$ at $8 \mathrm{TeV}$. In the figure we neglect the effect of higher-dimensional operators on the branching ratios.

2. attach momentum-dependent form factors to soften the ultraviolet behavior. The main problem is that after going through a lot of trouble of defining an effective field theory hypothesis, we spoil it by introducing ad-hoc non-local interactions in the position-space Lagrangian.

3. only use data in phase space regions which are not sensitive to the ultraviolet completions, for example requiring $p_{T}<100 \mathrm{GeV}$ for the tagging jets in weak boson fusion [124-128] or an upper bound on $p_{T}^{V}$ in $V H$ production. The obvious disadvantage of this approach is that we lose experimental information and produce worse bounds, as we will see.

In this analysis we will attempt to include as much of the kinematic information as possible, but carefully check how much of the distinguishing power comes from phase space regions not obviously consistently described by the effective field theory.

We start with $V H \rightarrow V(b \bar{b})$ production. To be maximally sensitive to $\mathcal{O}_{W W}, \mathcal{O}_{B B}$, $\mathcal{O}_{W}$, and $\mathcal{O}_{B}$ requires a kinematic variable with large flow through the production vertex. A key candidate is the transverse momentum distribution in the hard process [47] of the cut-based experimental analysis, which serves as a check of the measured rate, while the 
measurement itself used in section 2 relies on a multi-variate analysis. The experimental search requires two jets with medium and tight $b$-tags and defines three categories with 0 , 1 and 2 leptons that receive contributions from $H W$ and $H Z$ productions, therefore being sensitive to different contributions of the higher-dimensional operators. We show these three distributions in figure 10 with a selection of dimension- 6 anomalous contributions. While the background rapidly decreases at large transverse momenta, the main effect of the dimension-6 operators is conversely an enhancement at high momenta, being most prominent in the last bin of the distributions.

To combine the different lepton multiplicities we use our FEynRules [119] implementation of the dimension-6 operators to generate the distributions with MADGRAPH5 [118], Pythia [129], and PGS4 [130], the latter checked with Delphes [131]. We use the SM Higgs expectations to calibrate our setup to the distributions and rates shown in ref. [47]. We parametrize the kinematic distributions as a function of the dimension-6 operators and we use the SM background expectations and the number of measured events per bin in ref. [47].

As mentioned above, the $p_{T}^{V}$ distributions shown in figure 10 correspond to a cutbased ATLAS analysis [47]. When adding this information to the global Run I analysis consistently we need to be careful: first, we cannot use the same information twice. This means we could remove the corresponding total rates from the analysis and instead include the binned distributions. However, the cut-based analysis is weaker than the multi-variate analysis and they do not give the same measured central values. This would render any estimate of the additional power of the kinematic information impossible. Instead, we keep the multi-variate rate information and add the kinematic information through a set of asymmetries based on the bin content of figure 10,

$$
A_{i}=\frac{\operatorname{bin}_{i+1}-\operatorname{bin}_{i}}{\operatorname{bin}_{i+1}+\operatorname{bin}_{i}}
$$

which for each leptonic channel defines three or four additional measurements.

Our second test case is the azimuthal angle correlation in weak boson fusion production with $H \rightarrow \gamma \gamma$ [132-136]. Because the measurement of $\Delta \phi_{j j}$ does not require the reconstruction of any reference frame, its uncertainties are reduced. Unfortunately, the corresponding distributions are not shown in the most prominent weak boson fusion channels with decays $H \rightarrow W^{+} W^{-}$and $H \rightarrow \tau^{+} \tau^{-}$. An unfolded distribution is in contrast available for the decay $H \rightarrow \gamma \gamma$ [60]. However, due to the lack of cuts on $m_{j j}$ and $\Delta \eta_{j j}$, the weak boson fusion mode accounts for less than $35 \%$ of all signal events, diluting consequently the promising power of the $\Delta \phi_{j j}$ variable in this production mechanism.

In the present absence of a better alternative we include the above channels in our SFITTER analysis. To simulate SM Higgs production in weak boson fusion and the $V H$ channel we rely on the same selection of tools we have used for the $p_{T}^{V}$ implementation. To validate our calculations, we compare our SM simulations to the ATLAS result, most notably the plots available in HEPDATA [137]. Once our setup is tested we simulate the effect of dimension- 6 operators on the weak boson fusion and $V H$ distributions. The main contribution from Higgs production in gluon fusion is only affected by $\mathcal{O}_{G G}, \mathcal{O}_{\Phi, 2}$, and 
$\mathcal{O}_{b, t}$, none of which change the Lorentz structure of the hard process. We can then use the central estimate by ATLAS, properly reweighted by the introduced shift of the relevant operators. We use a similar reweighting to simulate the effects of the effective operators in the di-photon decay, as none of the operators generate a non-SM Lorentz structure for this vertex either. In the lower right panel of figure 10 we show the $\Delta \phi_{j j}$ distribution with a selection of dimension-6 contributions. It turns out that all $\mathcal{O}_{W W}, \mathcal{O}_{B B}, \mathcal{O}_{W}$, and $\mathcal{O}_{B}$ peak at 0 or at $\pi[132-136]$.

To add $\Delta \phi_{j j}$ to the global analysis we again keep the measured total rates used in the previous analyses and construct three additional asymmetries [132-136],

$$
\begin{aligned}
& A_{1}= \frac{\sigma\left(\Delta \phi_{j j}<\frac{\pi}{3}\right)+\sigma\left(\Delta \phi_{j j}>\frac{2 \pi}{3}\right)-\sigma\left(\frac{\pi}{3}<\Delta \phi_{j j}<\frac{2 \pi}{3}\right)}{\sigma\left(\Delta \phi_{j j}<\frac{\pi}{3}\right)+\sigma\left(\Delta \phi_{j j}>\frac{2 \pi}{3}\right)+\sigma\left(\frac{\pi}{3}<\Delta \phi_{j j}<\frac{2 \pi}{3}\right)}, \\
& A_{2}= \frac{\sigma\left(\Delta \phi_{j j}>\frac{2 \pi}{3}\right)-\sigma\left(\Delta \phi_{j j}<\frac{\pi}{3}\right)}{\sigma\left(\Delta \phi_{j j}>\frac{2 \pi}{3}\right)+\sigma\left(\Delta \phi_{j j}<\frac{\pi}{3}\right)}, \\
& A_{3}=\frac{\sigma\left(\Delta \phi_{j j}>\frac{5 \pi}{6}\right)-\sigma\left(\frac{2 \pi}{3}<\Delta \phi_{j j}<\frac{5 \pi}{6}\right)}{\sigma\left(\Delta \phi_{j j}>\frac{5 \pi}{6}\right)+\sigma\left(\frac{2 \pi}{3}<\Delta \phi_{j j}<\frac{5 \pi}{6}\right)} .
\end{aligned}
$$

The first asymmetry is tailored to discriminate different production modes and $C P$ structures $[60,132-136]$. The second asymmetry enhances the sensitivity to $\mathcal{O}_{W W}, \mathcal{O}_{B B}, \mathcal{O}_{W}$, and $\mathcal{O}_{B}$ in weak boson fusion, all of which generate non-zero values for $A_{2}$. Finally, the third asymmetry is orthogonal to the other two, to not exclude any information.

\subsection{Full dimension-6 analysis}

The final step in our higher-dimensional SFiTTER analysis is to add these test distributions to the coupling information used in section 3.2. As we have discussed, we use the experimental information shown in figure $10[47,60]$ in terms of the asymmetries defined in eqs. (3.9) and (3.10).

The main technical problem of the purely rate-based analysis of dimension- 6 operators are the correlations which make it hard to extract 1-dimensional profile likelihoods and error bars for the individual $f_{x} / \Lambda^{2}$, as illustrated in figure 8 . We show the effect of kinematic distributions on some critical 2-dimensional profile likelihoods in figure 11. In the top row we show the results after including the $\Delta \phi_{j j}$ distribution only. Compared to the figure 8 we see very small improvement, except for a slight reduction of the secondary structure in the $\mathcal{O}_{B B}$ vs $\mathcal{O}_{B}$ and $\mathcal{O}_{W}$ vs $\mathcal{O}_{B}$ correlations. However, this reduced impact should not be taken as a statement about the distinguishing power of the $\Delta \phi_{j j}$ distribution; it is really linked to the lack of publicly available information on this distribution, as discussed above.

In the second row of figure 11 we show the impact of also adding the full $p_{T}^{V}$ information in $V H$ production. It significantly improves the situation with secondary solutions, largely removing the correlated structure for example in $\mathcal{O}_{W}$ vs $\mathcal{O}_{B}$. There still exists a weak secondary minimum for example in the $\mathcal{O}_{B B}$ vs $\mathcal{O}_{B}$ correlation, but because of its relative weakness it will allow us to derive a more straightforward 1-dimensional profile likelihood and an associated $68 \%$ CL error bar for example on $f_{B B} / \Lambda^{2}$. The reduction of the allowed space is notorious in the three corresponding panels in figure 11. Actually, the left panel 

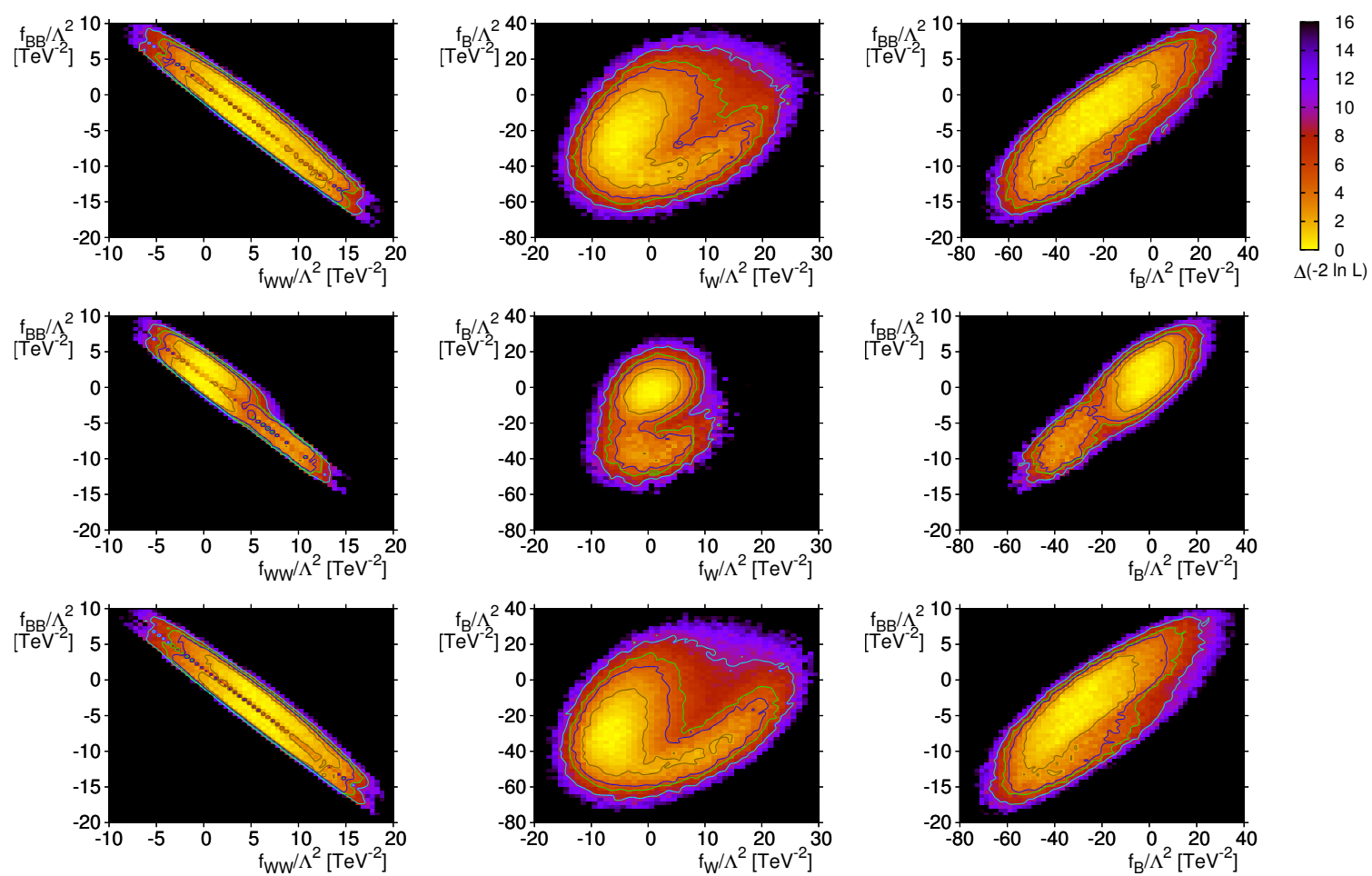

Figure 11. Correlations between different coefficients $f_{x} / \Lambda^{2}$ (measured in $\mathrm{TeV}^{-2}$ ) after including kinematic distributions. In the top row we add the $\Delta \phi_{j j}$ distribution; in the second row we also include $p_{T}^{V}$ from $V H$ production; in the bottom row we then remove the highest bin associated with large momentum flow through the dimension- 6 vertex. The 1-dimensional profile likelihoods of the second row correspond to the results shown as the blue bars in figure 12 .

shows that after including the $p_{T}^{V}$ and $\Delta \phi_{j j}$ distributions the fit becomes more sensitive to $\mathcal{O}_{W W}$ and $\mathcal{O}_{B B}$ through their individual contributions to the $H V V$ couplings mediating associated $V H$ production and weak boson fusion.

Finally, we need to check the consistency of the effective theory approach [138-140]. Based on Run I data our analysis typically probes $\left|f_{x} / \Lambda^{2}\right| \sim 10 / \mathrm{TeV}^{2}$. A hypothetical setting of $f_{x}$ to unity would correspond to new physics scales around $\Lambda \sim 300 \mathrm{GeV}$. According to figure 10 the highest momentum bin of the $p_{T}^{V}$ distribution starts from $p_{T}^{V}=200 \mathrm{GeV}$ and includes all events above this value. A conservative approach would be to exclude this last bin, and thus the last asymmetries defined in eq. (3.9), from the kinematic analysis. In the bottom row of figure 11 we show the corresponding 2-dimensional correlations from this analysis. A comparison to the first row shows that almost the entire additional information of the $p_{T}^{V}$ distribution is encoded in the last bin. In the remainder of the discussion we will not follow this conservative approach, so it should be noted that the full SFITTER analysis of the higher-dimensional operators has to be taken with a grain of salt. On the other hand, the analysis including kinematic distributions is mostly meant to be a proof of principle, and the consistency of the Higgs effective theory will clearly improve with Run II data.

With this in mind we show the best fit points and the corresponding 1-dimensional $68 \%$ CL error bars including kinematic distributions in figure 12. In contrast to figure 9 we do 


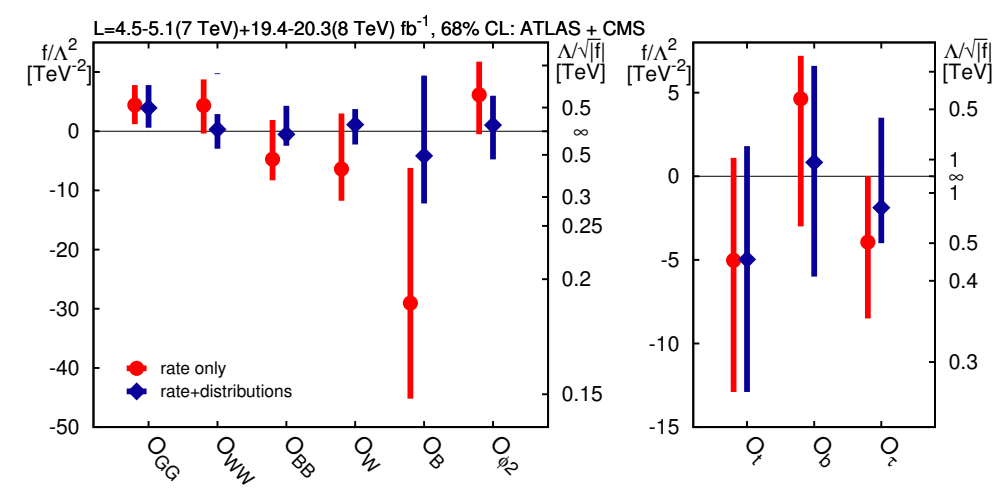

Figure 12. $68 \%$ CL error bars on the Wilson coefficients $f_{x} / \Lambda^{2}$ for the dimension-6 operators defined in eq. (3.3) (left panel) and eq. (3.7) (right panel). In addition to total rate information we also include kinematic distributions and only show $68 \%$ CL contours. For the Yukawa couplings as well as for $\mathcal{O}_{G G}$ we limit ourselves to the SM-like solution for this representation.

not show secondary solutions for the signs of the Yukawa-like couplings and for $\mathcal{O}_{G G}$. We also limit ourselves to $68 \%$ CL contours. We see that $\mathcal{O}_{B}$ and $\mathcal{O}_{W}$ are the operators most affected by the addition of kinematic distributions, closely followed by $\mathcal{O}_{W W}$ and $\mathcal{O}_{B B}$. Typical energy scales probed by Run I data are $300 \mathrm{GeV}$ to $500 \mathrm{GeV}$ if order one Wilson coefficients are assumed, with less significant constraints in the fermion sector. All coefficients are in agreement with zero, and the one to two sigma deviations are hard to map onto individual measurements. Including all available kinematic information visibly stabilizes the constraint on $f_{B}$ and moves every single best-fit point closer to the Standard Model prediction.

\section{Future: off-shell measurements}

ATLAS and CMS recently published a study on the contribution of Higgs exchange to $Z Z$ production at invariant masses well above the Higgs pole $m_{Z Z} \sim m_{H}$ [31-33, 61, 62]. Given the small Higgs width, such a measurement would normally only show a very moderate dependence on the Higgs mass. However, the kinematic structure of this particular channel turns it into a sensitive measurement. Approximately $\mathcal{O}(15 \%)$ of the rate mediated by the $s$-channel Higgs exchange lies in the off-shell regime, $m_{4 \ell}>130 \mathrm{GeV}$. In addition the leading effect arises from the signal interference with the continuum background. Some representative Feynman diagrams to this process are
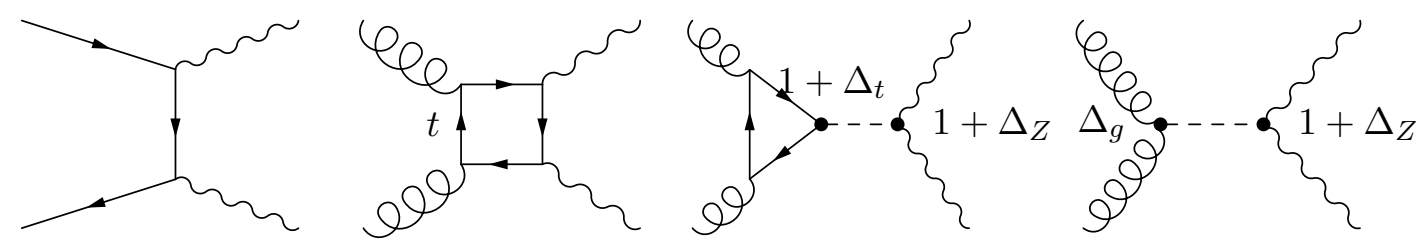

Note that $\Delta_{g}$ in this representation shows a non-trivial momentum dependence, limiting the model-independent features of the width measurement [34-38]. If the Higgs propagator in the interference is probed far above the mass shell, it behaves like $1 / s$. On-shell 
and off-shell Higgs rates then scale like

$$
\sigma_{i \rightarrow H \rightarrow f}^{\text {on-shell }} \propto \frac{g_{i}^{2}\left(m_{H}\right) g_{f}^{2}\left(m_{H}\right)}{\Gamma_{H}} \quad \quad \text { vs } \quad \quad \sigma_{i \rightarrow H^{*} \rightarrow f}^{\text {off-shell }} \propto g_{i}^{2}\left(m_{4 \ell}\right) g_{f}^{2}\left(m_{4 \ell}\right)
$$

where $g_{i}\left(g_{f}\right)$ refer to the Higgs couplings involved in the production (decay) for the present channel. Eventually, we will remove the assumptions about the Higgs width described in section 1.2 from the SFiTTER setup and instead determine the total width from the combination of off-shell and on-shell measurements. The Lagrangian of the underlying hypothesis reads

$$
\begin{aligned}
\mathcal{L}= & \mathcal{L}_{\mathrm{SM}}+\Delta_{W} g m_{W} H W^{\mu} W_{\mu}+\Delta_{Z} \frac{g}{2 c_{w}} m_{Z} H Z^{\mu} Z_{\mu}-\sum_{\tau, b, t} \Delta_{f} \frac{m_{f}}{v} H\left(\bar{f}_{R} f_{L}+\text { h.c. }\right) \\
& +\Delta_{g} F_{G} \frac{H}{v} G_{\mu \nu} G^{\mu \nu}+\Delta_{\gamma} F_{A} \frac{H}{v} A_{\mu \nu} A^{\mu \nu}+\text { invisible decays + unobservable decays } .
\end{aligned}
$$

The distinction between the two terms linked to decays to non-SM states is that 'invisible decays' are reconstructable using missing transverse momentum, while 'unobservable decays' are for some other reason not observable at the LHC, for example because of overwhelming jet backgrounds [10]. Not accounting for such unobservable decays would lead to shifts of all $\Delta_{x}$ as compared to the analysis including these decays.

Before we allow for a fully unconstrained Higgs width through unobservable decay channels we combine on-shell and off-shell analysis to probe the energy dependence of the operators involved [141]. On the Higgs production side, the dimension-6 operators entering the off-shell measurements are listed in eqs. (3.3) and (3.7), namely $\mathcal{O}_{G G}, \mathcal{O}_{\Phi, 2}$ and $\mathcal{O}_{t, b}$. They can be described by the two parameters $\Delta_{g}$ and $\Delta_{t}$ or equivalently $g_{H g g}$ and $g_{f}$ (with a marginal contribution from the bottom loop). The difference between the two are top mass effects in the kinematic structure [87]. In the decay the dimension-6 operators in eq. (3.4) lead to additional operator structures, namely $Z_{\mu \nu} Z^{\mu} \partial^{\nu} H$ and $H Z_{\mu \nu} Z^{\mu \nu}$ in eq. (3.4). None of them affects the longitudinal $Z$-polarization [142], so they lead to similar $m_{4 \ell}$ kinematics as the SM-operator $H Z_{\mu} Z^{\mu}$. We parametrize this Higgs decay only accounting for $\Delta_{Z}$ or $g_{H Z Z}^{(3)}$. Properly accounting for the continuum background we can write the gluon fusion component to the signal as

$$
\begin{aligned}
\mathcal{M}_{g g \rightarrow Z Z}= & \left(1+\Delta_{Z}\right)\left[\left(1+\Delta_{t}\right) \mathcal{M}_{t}+\Delta_{g} \mathcal{M}_{g}\right]+\mathcal{M}_{c} \\
\frac{d \sigma}{d m_{4 \ell}}= & \left(1+\Delta_{Z}\right)\left[\left(1+\Delta_{t}\right) \frac{d \sigma_{t c}}{d m_{4 \ell}}+\Delta_{g} \frac{d \sigma_{g c}}{d m_{4 \ell}}\right] \\
& +\left(1+\Delta_{Z}\right)^{2}\left[\left(1+\Delta_{t}\right)^{2} \frac{d \sigma_{t t}}{d m_{4 \ell}}+\left(1+\Delta_{t}\right) \Delta_{g} \frac{d \sigma_{t g}}{d m_{4 \ell}}+\Delta_{g}^{2} \frac{d \sigma_{g g}}{d m_{4 \ell}}\right]+\frac{d \sigma_{c}}{d m_{4 \ell}} .
\end{aligned}
$$

We illustrate the top mass effects in figure 13. The background $q \bar{q} \rightarrow Z Z$ and the data points are taken from the experimental publications [61, 62]. The gluon-initiated component is generated with MCFM [143] following eq. (4.3). QCD corrections to the gluon-induced component are accounted via a global $K$-factor [31-33, 144]. We follow the ATLAS and CMS cut-flow and find full agreement with both studies. 

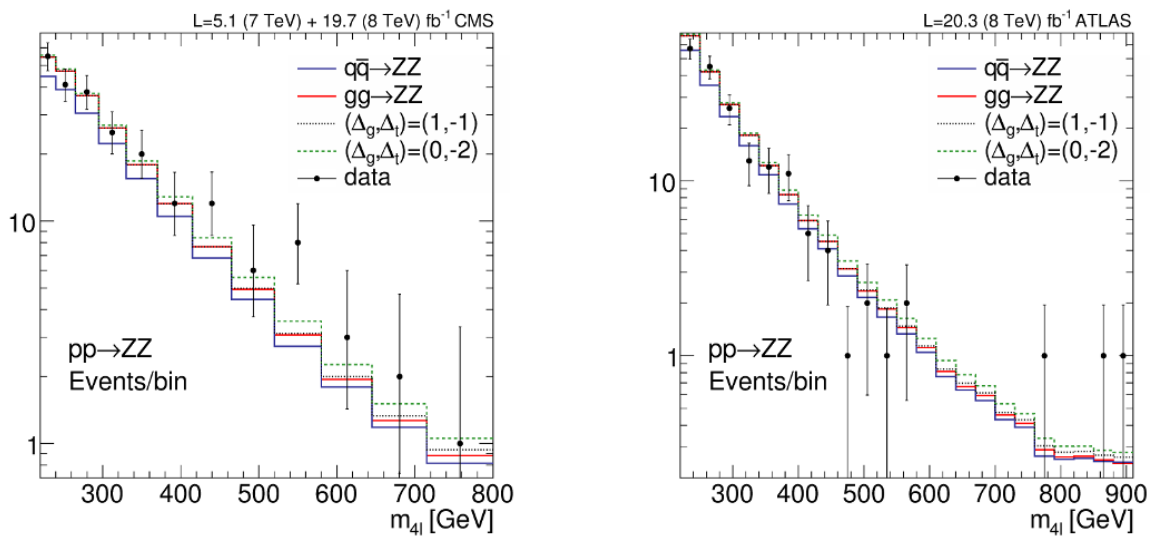

Figure 13. $m_{4 \ell}$ distribution for the CMS (left) and ATLAS (right) analyses. The $q \bar{q} \rightarrow Z Z$ background and the data points are obtained from refs. [61, 62]. The remaining curves are generated following the parametrization of eq. (4.3).
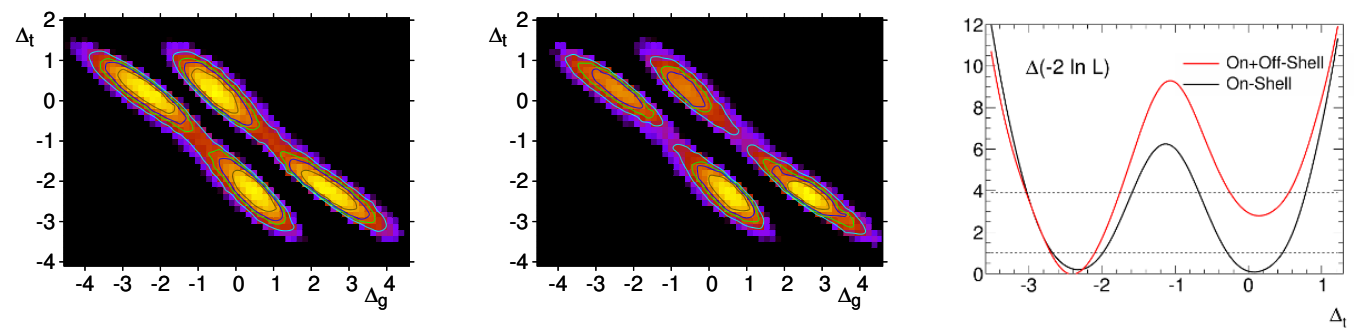

Figure 14. Correlation between the coupling modifications $\Delta_{t}$ and $\Delta_{g}$ without (left) and with (center) off-shell Higgs measurements. In the right panel we show the 1-dimensional profile likelihood for $\Delta_{t}$ with and without off-shell measurements.

Following eq. (4.3), terms linear in $\left(1+\Delta_{t}\right)$ are sensitive to the sign of the top Yukawa coupling [87]: in the Standard Model the off-shell interference is destructive, while a sign change in the top Yukawa coupling increases the combined rate significantly. In addition, we see the kinematic difference from the missing top mass threshold and the missing logarithmic top mass dependence for the dimension- 6 operator. Similar top mass effects can be observed in gluon fusion Higgs production with hard jets [87, 145-149] and in the gluoninduced contribution to $V H$ production [150]. Both should eventually be included in the Higgs couplings analysis.

One way to exploit this feature in our Higgs couplings determination is to include the usual coupling modifications and an invisible branching ratio, but no unobservable width. In this case we probe the momentum dependence of the effective Higgs-gluon coupling, linked to its top mass dependence [87]. In figure 14 we present the resulting correlation between $\Delta_{t}$ and $\Delta_{g}$, finding a significant improvement from the off-shell rate measurement. The SM-like and flipped-sign solutions clearly separate. In the right panel of figure 14 we observe a slight preference towards a negative top Yukawa coupling. It arises from a small excess of events in the off-shell CMS data. ATLAS sees the opposite trend, but with considerably fewer events. 

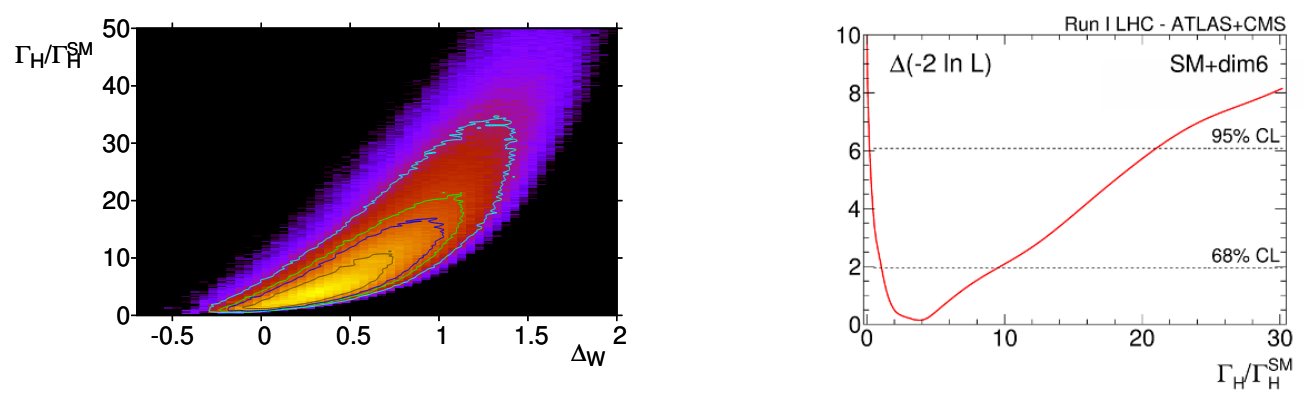

Figure 15. Correlation between the total Higgs width and the coupling modifications $\Delta_{W}$ (left) and 1-dimensional profile likelihood of the total Higgs width (right). Both, on-shell and off-shell Higgs rates are included.

Finally, we can allow for unobserved decays according to eq. (4.2). This corresponds to a free total Higgs width under the condition that the minimum width is given by the sum of the observed partial widths. In figure 15, we present first results from a global SFITTER analysis. In the left panel we show the correlation between a typical coupling $\Delta_{W}$ and the total Higgs width. For $\Gamma_{H} / \Gamma_{H}^{\mathrm{SM}} \gg 1$ the Higgs production and decay rates scale like $g_{x}^{4} / \Gamma_{H}$. Indeed, we see that the positive correlation extends to $\Gamma_{H} / \Gamma_{H}^{\mathrm{SM}} \sim 30 \sim 2.3^{4}$. The corresponding value of $\Delta_{W}$ is then 1.3 , just as expected.

In the right panels we see that the upper bound is approximately $\Gamma_{H}<9.3 \Gamma_{H}^{\mathrm{SM}}$ at $68 \%$ CL. While our width constraint was obtained in the EFT context, our bound is still competitive to other analysis that account only to SM-like interactions [31-33]. The key ingredient here is the analysis of the whole $m_{4 l}$ distribution profile that probes the possible new physics at different energy scales. A similar study via on-shell signal strengths would clearly not be as sensitive.

\section{$5 \quad$ Present and future}

In this paper we have presented a final analysis of the Run I Higgs measurements. For the first time, we directly compare the direct Higgs coupling analysis with the effective Lagrangian approach. For the coupling analysis we included independent variations of the Higgs-top and Higgs-gluon coupling, as well as an invisible Higgs branching ratio and eventually Higgs decays through unobserved channels. While for the former the current results for $t \bar{t} H$ production should be taken with a grain of salt, we found a stable combined measurement of $\mathrm{BR}_{\mathrm{inv}}=0.16_{-0.11}^{+0.07}$. Including off-shell Higgs measurements the full couplings fit gave an upper limit on the total Higgs width of $\Gamma_{H}<9.3 \Gamma_{H}^{\mathrm{SM}}$ at $68 \%$ CL.

Theoretical uncertainties and their effect on the individual error bars of more fundamental parameters are starting to become a crucial issue already by the end of Run I. We tested different assumptions on the correlation of theoretical uncertainties, on their size, and on their statistical treatment. As in the absence of a clear definition based on statistical principles we at least ensured that our analysis was conservative. Based on our findings the issue of theoretical uncertainties and their statistical treatment needs to be carefully considered for the upcoming Run II data. 
To include kinematic distributions the traditional coupling analysis has to be expanded. We consider an effective field theory approach the most natural and the most promising expansion. As long as we only include Higgs rate information the Run I analysis in terms of Higgs coupling modifications $\Delta_{x}$ and in terms of higher-dimensional operators $f_{x} / \Lambda^{2}$ are essentially equivalent. Once we included measurements from the pure gauge sector this will change. Technically, the rate-based analysis in terms of an effective field theory is more challenging, because the underlying parameters are less directly linked to measurements than for the Higgs coupling modifications.

We added two sample distributions from $V H$ production and from weak boson fusion to our analysis. We found a significant stabilization of the higher-dimensional analysis, the secondary structures were more easily identified, and all individual error bars on the Wilson coefficients were visibly reduced. We end the discussion with a word of warning: given the current precision in the dimension- 6 analysis it is not guaranteed that the effective Lagrangian expansion is within its range of validity for all considered observables. It should then be seen as a motivated and useful parametrization of Higgs interactions, while we wait for the increase of precision in the future Run II of the LHC.

\section{Acknowledgments}

All of us are very grateful to Concha Gonzalez-Garcia's support during all stages of this project. Not only would this paper look very different without her input, the project would never even have started without her physics contribution and her energy. Moreover, we are grateful to the SFiTTER team with Dirk Zerwas, Markus Klute, and Rémi Lafaye who continuously keep our project alive.

O.J.P.E. is supported in part by Conselho Nacional de Desenvolvimento Científico e Tecnológico $(\mathrm{CNPq})$ and by Fundação de Amparo à Pesquisa do Estado de São Paulo (FAPESP), T.C is supported by U.S.A.-NSF grant PHY-09-6739. We also thank support from EU grant FP7 ITN INVISIBLES (Marie Curie Actions PITN-GA-2011-289442).

\section{A Numerical results}

In this appendix we give the limits presented in the figures throughout the paper as numbers. 


\begin{tabular}{|l|c|c|c|c|c|c|}
\cline { 2 - 7 } \multicolumn{1}{c|}{} & \multicolumn{4}{c|}{5 parameter analysis } & \multicolumn{4}{c|}{6 parameter analysis } \\
\cline { 2 - 7 } \multicolumn{1}{c|}{} & Best fit & $68 \%$ CL intervals & $95 \%$ CL intervals & Best fit & $68 \%$ CL intervals & $95 \%$ CL intervals \\
\hline$\Delta_{W}$ & -0.113 & $(-0.23,0.02)$ & $(-0.335,0.145)$ & -0.185 & $(-0.35,-0.05)$ & $(-0.465,0.095)$ \\
\hline$\Delta_{Z}$ & 0.0563 & $(-0.07,0.18)$ & $(-0.20,0.295)$ & 0.041 & $(-0.08,0.175)$ & $(-0.22,0.285)$ \\
\hline$\Delta_{t}$ & -0.271 & $(-0.38,-0.08)$ & $(-0.495,0.09)$ & -0.271 & $(-0.41,-0.08)$ & $(-0.53,0.09)$ \\
\hline$\Delta_{b}$ & -0.291 & $(-0.535,0.035)$ & $(-0.785,0.36)$ & -0.304 & $(-0.57,0.015)$ & $(-0.83,0.355)$ \\
\hline$\Delta_{\tau}$ & -0.0987 & $(-0.265,0.095)$ & $(-0.4,0.26)$ & -0.0826 & $(-0.265,0.095)$ & $(-0.405,0.29)$ \\
\hline$\Delta_{\gamma}$ & - & - & - & 0.129 & $(0.015,0.29)$ & $(-0.11,0.42)$ \\
\hline$\Delta_{\gamma}^{\mathrm{SM}+\mathrm{NP}}$ & - & - & - & -0.033 & $(-0.175,0.13)$ & $(-0.305,0.31)$ \\
\hline
\end{tabular}

Table 1. Best fit values, $68 \% \mathrm{CL}$ and $95 \% \mathrm{CL}$ allowed ranges for the results of the Higgs analysis with 5 free couplings (blue bars in figure 1) and the results of the analysis including in addition $\Delta_{\gamma}$ (blue bars in the left panel in figure 2).

\begin{tabular}{|l|c|c|c|c|c|c|}
\cline { 2 - 7 } \multicolumn{1}{c|}{} & \multicolumn{4}{|c|}{7 parameter analysis } & \multicolumn{4}{c|}{8 parameter analysis } \\
\cline { 2 - 7 } \multicolumn{1}{c|}{} & Best fit & $68 \%$ CL intervals & $95 \%$ CL intervals & Best fit & $68 \%$ CL intervals & 95\% CL intervals \\
\hline$\Delta_{W}$ & -0.160 & $(-0.335,-0.05)$ & $(-0.46,0.085)$ & -0.0867 & $(-0.265,0.025)$ & $(-0.38,0.155)$ \\
\hline$\Delta_{Z}$ & 0.0559 & $(-0.07,0.195)$ & $(-0.205,0.305)$ & 0.158 & $(0.01,0.28)$ & $(-0.125,0.405)$ \\
\hline$\Delta_{t}$ & 0.159 & $(-0.2,0.46)$ & $(-0.585,0.75)$ & 0.188 & $(-0.13,0.57)$ & $(-0.505,0.845)$ \\
\hline$\Delta_{b}$ & -0.265 & $(-0.565,-0.01)$ & $(-0.82,0.295)$ & -0.193 & $(-0.5,0.06)$ & $(-0.77,0.375)$ \\
\hline$\Delta_{\tau}$ & -0.0492 & $(-0.25,0.095)$ & $(-0.395,0.28)$ & 0.0417 & $(-0.17,0.185)$ & $(-0.33,0.375)$ \\
\hline$\Delta_{\gamma}$ & 0.226 & $(0.09,0.40)$ & $(-0.065,0.555)$ & 0.248 & $(0.1,0.435)$ & $(-0.055,0.595)$ \\
\hline$\Delta_{g}$ & -0.479 & $(-0.83,-0.125)$ & $(-1,0.37)$ & -0.430 & $(-0.855,-0.13)$ & $(-1,0.385)$ \\
\hline BR $R_{\text {inv }}$ & - & - & - & 0.157 & $(0.048,0.226)$ & $(0 ., 0.306)$ \\
\hline$\Delta_{\gamma}^{\mathrm{SM}+\mathrm{NP}}$ & -0.0191 & $(-0.17,0.125)$ & $(-0.295,0.285)$ & 0.0892 & $(-0.09,0.22)$ & $(-0.22,0.395)$ \\
\hline$\Delta_{g}^{\mathrm{SM}+\mathrm{NP}}$ & 0.230 & $(-0.4,0.115)$ & $(-0.51,0.35)$ & -0.163 & $(-0.335,0.04)$ & $(-0.45,0.115)$ \\
\hline
\end{tabular}

Table 2. Best fit values, $68 \% \mathrm{CL}$ and $95 \% \mathrm{CL}$ allowed ranges for the results of the analysis with 7 free couplings (blue bars in the right panel in figure 2) and the results of the analysis including in addition $\mathrm{BR}_{\text {inv }}$ (blue bars in figure 4 ). 


\begin{tabular}{|l|c|c|c|c|c|c|}
\cline { 2 - 7 } \multicolumn{1}{c|}{} & \multicolumn{3}{c|}{ Correlated theoretical uncertainties } & \multicolumn{3}{c|}{$\mathrm{N}^{3}$ LO gluon fusion prediction } \\
\cline { 2 - 7 } \multicolumn{1}{c|}{} & Best fit & $68 \%$ CL intervals & $95 \%$ CL intervals & Best fit & $68 \%$ CL intervals & $95 \%$ CL intervals \\
\hline$\Delta_{W}$ & -0.137 & $(-0.26,-0.005)$ & $(-0.39,0.115)$ & -0.162 & $(-0.315,-0.04)$ & $(-0.44,0.09)$ \\
\hline$\Delta_{Z}$ & 0.0814 & $(-0.02,0.225)$ & $(-0.14,0.335)$ & 0.0704 & $(-0.07,0.19)$ & $(-0.205,0.295)$ \\
\hline$\Delta_{t}$ & 0.113 & $(-0.07,0.525)$ & $(-0.315,0.805)$ & 0.165 & $(-0.195,0.475)$ & $(-0.565,0.76)$ \\
\hline$\Delta_{b}$ & -0.317 & $(-0.51,-0.01)$ & $(-0.725,0.28)$ & -0.271 & $(-0.55,0.005)$ & $(-0.8,0.295)$ \\
\hline$\Delta_{\tau}$ & -0.043 & $(-0.195,0.105)$ & $(-0.32,0.27)$ & -0.0939 & $(-0.25,0.085)$ & $(-0.395,0.26)$ \\
\hline$\Delta_{\gamma}$ & 0.213 & $(0.105,0.375)$ & $(-0.015,0.52)$ & 0.241 & $(0.09,0.395)$ & $(-0.06,0.54)$ \\
\hline$\Delta_{g}$ & -0.386 & $(-0.85,-0.215)$ & $(-1 ., 0.08)$ & -0.508 & $(-0.82,-0.11)$ & $(-1 ., 0.39)$ \\
\hline$\Delta_{\gamma}^{\mathrm{SM}+\mathrm{NP}}$ & 0.0088 & $(-0.115,0.15)$ & $(-0.23,0.29)$ & -0.00786 & $(-0.16,0.12)$ & $(-0.28,0.28)$ \\
\hline$\Delta_{g}^{\mathrm{SM}+\mathrm{NP}}$ & -0.195 & $(-0.355,-0.055)$ & $(-0.45,0.11)$ & -0.248 & $(0.37,-0.095)$ & $(-0.49,0.045)$ \\
\hline
\end{tabular}

Table 3. Best fit values, 68\% CL and 95\% CL allowed ranges for the results of the analysis with 7 free couplings assuming correlated theoretical uncertainties (light blue bars in the upper-left panel in figure 7) and the results of the analysis including $\mathrm{N}^{3} \mathrm{LO}$ corrections to the Higgs gluon fusion production rate at the LHC [96] (light blue bars in lower-left panel in figure 7).

\begin{tabular}{|l|c|c|c|c|c|c|}
\cline { 2 - 7 } \multicolumn{1}{c|}{} & \multicolumn{3}{c|}{ Passarino gluon fusion prediction } & \multicolumn{4}{c|}{ Gaussian theoretical errors } \\
\cline { 2 - 7 } \multicolumn{1}{c|}{} & Best fit & $68 \%$ CL intervals & $95 \%$ CL intervals & Best fit & $68 \%$ CL intervals & 95\% CL intervals \\
\hline$\Delta_{W}$ & -0.174 & $(-0.355,-0.06)$ & $(-0.47,0.09)$ & -0.118 & $(-0.295,0.01)$ & $(-0.425,0.14)$ \\
\hline$\Delta_{Z}$ & 0.0653 & $(-0.08,0.19)$ & $(-0.21,0.305)$ & -0.03848 & $(-0.16,0.105)$ & $(-0.3,0.21)$ \\
\hline$\Delta_{t}$ & 0.139 & $(-0.205,0.445)$ & $(-0.585,0.7)$ & 0.188 & $(-0.235,0.535)$ & $(-0.835,0.735)$ \\
\hline$\Delta_{b}$ & -0.291 & $(-0.58,-0.015)$ & $(-0.845,0.31)$ & -0.151 & $(-0.48,0.18)$ & $(-0.78,0.585)$ \\
\hline$\Delta_{\tau}$ & -0.0674 & $(-0.265,0.09)$ & $(-0.415,0.295)$ & -0.0700 & $(-0.255,0.1)$ & $(-0.405,0.305)$ \\
\hline$\Delta_{\gamma}$ & 0.231 & $(0.085,0.4)$ & $(-0.065,0.56)$ & 0.150 & $(0 ., 0.315)$ & $(-0.24,0.45)$ \\
\hline$\Delta_{g}$ & -0.552 & $(-0.895,-0.205)$ & $(-1 ., 0.305)$ & -0.372 & $(-0.755,-0.16)$ & $(-0.96,0.97)$ \\
\hline$\Delta_{\gamma}^{\mathrm{SM}+\mathrm{NP}}$ & -0.0266 & $(-0.19,0.115)$ & $(-0.305,0.305)$ & -0.0497 & $(-0.215,0.09)$ & $(-0.36,0.25)$ \\
\hline$\Delta_{g}^{\mathrm{SM}+\mathrm{NP}}$ & -0.309 & $(-0.455,-0.195)$ & $(-0.55,-0.045)$ & -0.117 & $(-0.28,0.05)$ & $(-0.415,0.255)$ \\
\hline
\end{tabular}

Table 4. Best fit values, $68 \%$ CL and 95\% CL allowed ranges for the results of the analysis with 7 free couplings using the gluon fusion prediction from ref. [95] (light blue bars in the upper-right panel in figure 7) and the results of the analysis assuming Gaussian theoretical uncertainties (light blue bars in lower-right panel in figure 7 ). 


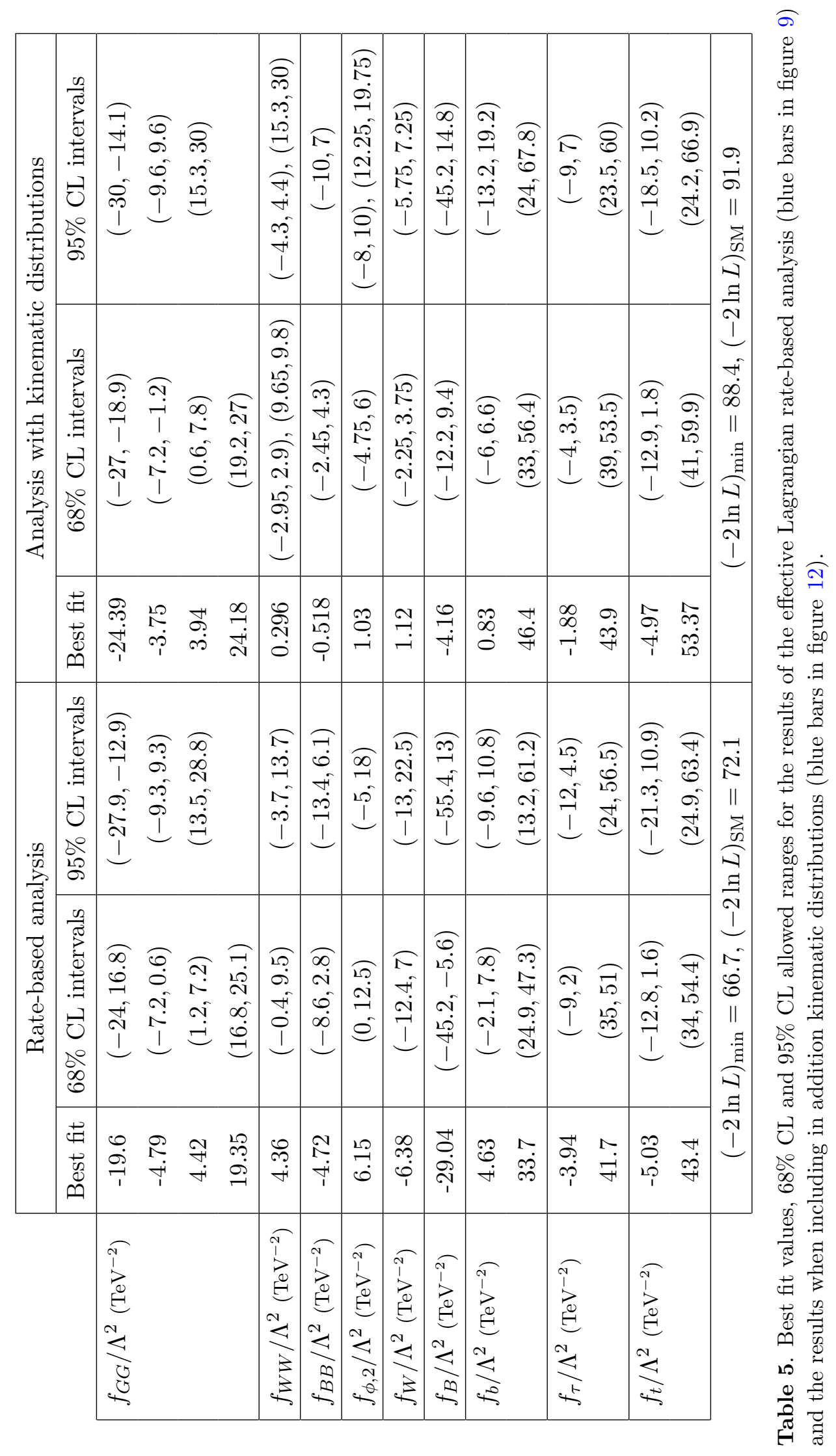


Open Access. This article is distributed under the terms of the Creative Commons Attribution License (CC-BY 4.0), which permits any use, distribution and reproduction in any medium, provided the original author(s) and source are credited.

\section{References}

[1] P.W. Higgs, Broken symmetries, massless particles and gauge fields, Phys. Lett. 12 (1964) 132 [INSPIRE].

[2] P.W. Higgs, Broken symmetries and the masses of gauge bosons, Phys. Rev. Lett. 13 (1964) 508 [INSPIRE].

[3] F. Englert and R. Brout, Broken symmetry and the mass of gauge vector mesons, Phys. Rev. Lett. 13 (1964) 321 [INSPIRE].

[4] ATLAS collaboration, Observation of a new particle in the search for the standard model Higgs boson with the ATLAS detector at the LHC, Phys. Lett. B 716 (2012) 1 [arXiv:1207.7214] [INSPIRE].

[5] CMS collaboration, Observation of a new boson at a mass of $125 \mathrm{GeV}$ with the CMS experiment at the LHC, Phys. Lett. B 716 (2012) 30 [arXiv:1207.7235] [INSPIRE].

[6] D.E. Morrissey, T. Plehn and T.M.P. Tait, Physics searches at the LHC, Phys. Rept. 515 (2012) 1 [arXiv:0912.3259] [INSPIRE].

[7] D. Zeppenfeld, R. Kinnunen, A. Nikitenko and E. Richter-Was, Measuring Higgs boson couplings at the CERN LHC, Phys. Rev. D 62 (2000) 013009 [hep-ph/0002036] [INSPIRE].

[8] M. Dührssen et al., Extracting Higgs boson couplings from CERN LHC data, Phys. Rev. D 70 (2004) 113009 [hep-ph/0406323] [INSPIRE].

[9] M. Dührssen, Prospects for the measurement of Higgs boson coupling parameters in the mass range from 110-190 GeV, ATL-PHYS-2003-030, CERN, Geneva Switzerland (2003).

[10] R. Lafaye, T. Plehn, M. Rauch, D. Zerwas and M. Dührssen, Measuring the Higgs sector, JHEP 08 (2009) 009 [arXiv:0904.3866] [InSPIRE].

[11] M. Klute, R. Lafaye, T. Plehn, M. Rauch and D. Zerwas, Measuring Higgs couplings from LHC data, Phys. Rev. Lett. 109 (2012) 101801 [arXiv:1205.2699] [INSPIRE].

[12] T. Plehn and M. Rauch, Higgs couplings after the discovery, Europhys. Lett. 100 (2012) 11002 [arXiv: 1207.6108] [INSPIRE].

[13] M. Klute, R. Lafaye, T. Plehn, M. Rauch and D. Zerwas, Measuring Higgs couplings at a linear collider, Europhys. Lett. 101 (2013) 51001 [arXiv:1301.1322] [INSPIRE].

[14] G. Bélanger, B. Dumont, U. Ellwanger, J.F. Gunion and S. Kraml, Global fit to Higgs signal strengths and couplings and implications for extended Higgs sectors, Phys. Rev. D 88 (2013) 075008 [arXiv: 1306.2941] [INSPIRE].

[15] P. Bechtle, S. Heinemeyer, O. Stål, T. Stefaniak and G. Weiglein, Probing the standard model with Higgs signal rates from the Tevatron, the LHC and a future ILC, JHEP 11 (2014) 039 [arXiv: 1403.1582] [INSPIRE].

[16] K. Cheung, J.S. Lee and P.-Y. Tseng, Higgs precision analysis updates 2014, Phys. Rev. D 90 (2014) 095009 [arXiv:1407.8236] [INSPIRE]. 
[17] J.-B. Flament, Higgs couplings and BSM physics: Run I legacy constraints, arXiv: 1504.07919 [INSPIRE].

[18] D. López-Val, T. Plehn and M. Rauch, Measuring extended Higgs sectors as a consistent free couplings model, JHEP 10 (2013) 134 [arXiv:1308.1979] [INSPIRE].

[19] T. Corbett, O.J.P. Eboli, J. Gonzalez-Fraile and M.C. Gonzalez-Garcia, Constraining anomalous Higgs interactions, Phys. Rev. D 86 (2012) 075013 [arXiv:1207.1344] [INSPIRE].

[20] T. Corbett, O.J.P. Eboli, J. Gonzalez-Fraile and M.C. Gonzalez-Garcia, Robust determination of the Higgs couplings: power to the data, Phys. Rev. D 87 (2013) 015022 [arXiv:1211.4580] [INSPIRE].

[21] T. Corbett, O.J.P. É boli, J. Gonzalez-Fraile and M.C. Gonzalez-Garcia, Determining triple gauge boson couplings from Higgs data, Phys. Rev. Lett. 111 (2013) 011801 [arXiv: 1304.1151] [INSPIRE].

[22] E. Massó and V. Sanz, Limits on anomalous couplings of the Higgs boson to electroweak gauge bosons from LEP and the LHC, Phys. Rev. D 87 (2013) 033001 [arXiv:1211.1320] [INSPIRE].

[23] B. Dumont, S. Fichet and G. von Gersdorff, A Bayesian view of the Higgs sector with higher dimensional operators, JHEP 07 (2013) 065 [arXiv: 1304.3369] [INSPIRE].

[24] S. Banerjee, S. Mukhopadhyay and B. Mukhopadhyaya, Higher dimensional operators and the LHC Higgs data: the role of modified kinematics, Phys. Rev. D 89 (2014) 053010 [arXiv: 1308.4860] [INSPIRE].

[25] I. Brivio et al., Disentangling a dynamical Higgs, JHEP 03 (2014) 024 [arXiv:1311.1823] [INSPIRE].

[26] J. Ellis, V. Sanz and T. You, Complete Higgs sector constraints on dimension-6 operators, JHEP 07 (2014) 036 [arXiv: 1404.3667] [INSPIRE].

[27] J. Ellis, V. Sanz and T. You, The effective standard model after LHC Run I, JHEP 03 (2015) 157 [arXiv: 1410.7703] [INSPIRE].

[28] T. Corbett, O. É boli and M. Gonzalez-Garcia, Unitarity constraints on dimension-6 operators, Phys. Rev. D 91 (2015) 035014 [arXiv:1411.5026] [INSPIRE].

[29] R. Edezhath, Dimension-6 operator constraints from boosted VBF Higgs, arXiv: 1501.00992 [INSPIRE].

[30] S. Banerjee, T. Mandal, B. Mellado and B. Mukhopadhyaya, Cornering dimension-6 HVV interactions at high luminosity LHC: the role of event ratios, arXiv:1505.00226 [INSPIRE].

[31] N. Kauer and G. Passarino, Inadequacy of zero-width approximation for a light Higgs boson signal, JHEP 08 (2012) 116 [arXiv:1206.4803] [INSPIRE].

[32] F. Caola and K. Melnikov, Constraining the Higgs boson width with $Z Z$ production at the LHC, Phys. Rev. D 88 (2013) 054024 [arXiv:1307.4935] [inSPIRE].

[33] J.M. Campbell, R.K. Ellis and C. Williams, Bounding the Higgs width at the LHC using full analytic results for $g g \rightarrow e^{-} e^{+} \mu^{-} \mu^{+}$, JHEP 04 (2014) 060 [arXiv:1311.3589] [INSPIRE].

[34] C. Englert and M. Spannowsky, Limitations and opportunities of off-shell coupling measurements, Phys. Rev. D 90 (2014) 053003 [arXiv: 1405.0285] [INSPIRE]. 
[35] J.S. Gainer, J. Lykken, K.T. Matchev, S. Mrenna and M. Park, Beyond geolocating: constraining higher dimensional operators in $H \rightarrow 4 \ell$ with off-shell production and more, Phys. Rev. D 91 (2015) 035011 [arXiv:1403.4951] [INSPIRE].

[36] G. Cacciapaglia, A. Deandrea, G. Drieu La Rochelle and J.-B. Flament, Higgs couplings: disentangling new physics with off-shell measurements, Phys. Rev. Lett. 113 (2014) 201802 [arXiv:1406.1757] [INSPIRE].

[37] C. Englert, Y. Soreq and M. Spannowsky, Off-shell Higgs coupling measurements in BSM scenarios, JHEP 05 (2015) 145 [arXiv:1410.5440] [INSPIRE].

[38] H.E. Logan, Hiding a Higgs width enhancement from off-shell $g g\left(\rightarrow h^{*}\right) \rightarrow Z Z$ measurements, arXiv:1412.7577 [INSPIRE].

[39] ATLAS collaboration, Observation and measurement of Higgs boson decays to $W W^{*}$ with the ATLAS detector, Phys. Rev. D 92 (2015) 012006 [arXiv:1412.2641] [INSPIRE].

[40] CMS collaboration, Measurement of Higgs boson production and properties in the $W W$ decay channel with leptonic final states, JHEP 01 (2014) 096 [arXiv:1312.1129] [INSPIRE].

[41] ATLAS collaboration, Measurements of Higgs boson production and couplings in the four-lepton channel in pp collisions at center-of-mass energies of 7 and $8 \mathrm{TeV}$ with the ATLAS detector, Phys. Rev. D 91 (2015) 012006 [arXiv:1408.5191] [InSPIRE].

[42] CMS collaboration, Measurement of the properties of a Higgs boson in the four-lepton final state, Phys. Rev. D 89 (2014) 092007 [arXiv:1312.5353] [INSPIRE].

[43] ATLAS collaboration, Measurement of Higgs boson production in the diphoton decay channel in pp collisions at center-of-mass energies of 7 and $8 \mathrm{TeV}$ with the ATLAS detector, Phys. Rev. D 90 (2014) 112015 [arXiv:1408.7084] [INSPIRE].

[44] CMS collaboration, Observation of the diphoton decay of the Higgs boson and measurement of its properties, Eur. Phys. J. C 74 (2014) 3076 [arXiv: 1407.0558] [INSPIRE].

[45] ATLAS collaboration, Evidence for the Higgs-boson Yukawa coupling to $\tau$ leptons with the ATLAS detector, JHEP 04 (2015) 117 [arXiv:1501.04943] [INSPIRE].

[46] CMS collaboration, Evidence for the $125 \mathrm{GeV}$ Higgs boson decaying to a pair of $\tau$ leptons, JHEP 05 (2014) 104 [arXiv:1401.5041] [InSPIRE].

[47] ATLAS collaboration, Search for the b $\bar{b}$ decay of the standard model Higgs boson in associated $(W / Z) H$ production with the ATLAS detector, JHEP 01 (2015) 069 [arXiv: 1409.6212] [INSPIRE].

[48] CMS collaboration, Search for the standard model Higgs boson produced in association with $a W$ or a $Z$ boson and decaying to bottom quarks, Phys. Rev. D 89 (2014) 012003 [arXiv:1310.3687] [INSPIRE].

[49] ATLAS collaboration, Search for the standard model Higgs boson in the $H \rightarrow Z \gamma$ decay mode with pp collisions at $\sqrt{s}=7$ and $8 \mathrm{TeV}$, ATLAS-CONF-2013-009, CERN, Geneva Switzerland (2013) [ATLAS-COM-CONF-2013-014].

[50] CMS collaboration, Search for a Higgs boson decaying into a $Z$ and a photon in pp collisions at $\sqrt{s}=7$ and $8 \mathrm{TeV}$, Phys. Lett. B 726 (2013) 587 [arXiv:1307.5515] [INSPIRE].

[51] ATLAS collaboration, Search for invisible decays of a Higgs boson produced in association with a Z boson in ATLAS, Phys. Rev. Lett. 112 (2014) 201802 [arXiv:1402.3244] [INSPIRE]. 
[52] ATLAS collaboration, Search for an invisibly decaying Higgs boson produced via vector boson fusion in pp collisions at $\sqrt{s}=8 \mathrm{TeV}$ using the ATLAS detector at the LHC, ATLAS-CONF-2015-004, CERN, Geneva Switzerland (2015) [ATLAS-COM-CONF-2015-004].

[53] ATLAS collaboration, Search for new phenomena in final states with an energetic jet and large missing transverse momentum in pp collisions at $\sqrt{s}=8 \mathrm{TeV}$ with the ATLAS detector, Eur. Phys. J. C 75 (2015) 299 [arXiv:1502.01518] [INSPIRE].

[54] ATLAS collaboration, Search for invisible decays of the Higgs boson produced in association with a hadronically decaying vector boson in pp collisions at $\sqrt{s}=8 \mathrm{TeV}$ with the ATLAS detector, Eur. Phys. J. C 75 (2015) 337 [arXiv:1504.04324] [INSPIRE].

[55] CMS collaboration, Search for invisible decays of Higgs bosons in the vector boson fusion and associated ZH production modes, Eur. Phys. J. C 74 (2014) 2980 [arXiv:1404.1344] [INSPIRE].

[56] CMS collaboration, Search for invisible decays of Higgs bosons in the vector boson fusion production mode, CMS-PAS-HIG-14-038, CERN, Geneva Switzerland (2014).

[57] ATLAS collaboration, Search for $H \rightarrow \gamma \gamma$ produced in association with top quarks and constraints on the Yukawa coupling between the top quark and the Higgs boson using data taken at $7 \mathrm{TeV}$ and $8 \mathrm{TeV}$ with the ATLAS detector, Phys. Lett. B 740 (2015) 222 [arXiv: 1409.3122] [INSPIRE].

[58] CMS collaboration, Search for the associated production of the Higgs boson with a top-quark pair, JHEP 09 (2014) 087 [Erratum ibid. 10 (2014) 106] [arXiv:1408.1682] [INSPIRE].

[59] CMS collaboration, Search for a standard model Higgs boson produced in association with a top-quark pair and decaying to bottom quarks using a matrix element method, Eur. Phys. J. C 75 (2015) 251 [arXiv:1502.02485] [INSPIRE].

[60] ATLAS collaboration, Measurements of fiducial and differential cross sections for Higgs boson production in the diphoton decay channel at $\sqrt{s}=8 \mathrm{TeV}$ with ATLAS, JHEP 09 (2014) 112 [arXiv: 1407.4222] [inSPIRE].

[61] ATLAS collaboration, Constraints on the off-shell Higgs boson signal strength in the high-mass $Z Z$ and $W W$ final states with the ATLAS detector, Eur. Phys. J. C 75 (2015) 335 [arXiv: 1503.01060] [INSPIRE].

[62] CMS collaboration, Constraints on the Higgs boson width from off-shell production and decay to Z-boson pairs, Phys. Lett. B 736 (2014) 64 [arXiv:1405.3455] [INSPIRE].

[63] R. Alonso, M.B. Gavela, L. Merlo, S. Rigolin and J. Yepes, The effective chiral Lagrangian for a light dynamical "Higgs particle", Phys. Lett. B 722 (2013) 330 [Erratum ibid. B 726 (2013) 926] [arXiv : 1212.3305] [INSPIRE].

[64] M.B. Gavela et al., CP violation with a dynamical Higgs, JHEP 10 (2014) 044 [arXiv:1406.6367] [INSPIRE].

[65] I. Brivio et al., Higgs ultraviolet softening, JHEP 12 (2014) 004 [arXiv:1405.5412] [INSPIRE].

[66] G. Buchalla, O. Catà and C. Krause, Complete electroweak chiral Lagrangian with a light Higgs at NLO, Nucl. Phys. B 880 (2014) 552 [arXiv:1307.5017] [INSPIRE].

[67] G. Buchalla, O. Catá and C. Krause, On the power counting in effective field theories, Phys. Lett. B 731 (2014) 80 [arXiv:1312.5624] [INSPIRE]. 
[68] G. Buchalla, O. Catà, A. Celis and C. Krause, Note on anomalous Higgs-boson couplings in effective field theory, arXiv:1504.01707 [INSPIRE].

[69] B. Patt and F. Wilczek, Higgs-field portal into hidden sectors, hep-ph/0605188 [INSPIRE].

[70] R. Schabinger and J.D. Wells, A minimal spontaneously broken hidden sector and its impact on Higgs boson physics at the Large Hadron Collider, Phys. Rev. D 72 (2005) 093007 [hep-ph/0509209] [INSPIRE].

[71] S. Bock et al., Measuring hidden Higgs and strongly-interacting Higgs scenarios, Phys. Lett. B 694 (2010) 44 [arXiv: 1007.2645] [INSPIRE].

[72] C. Englert, T. Plehn, D. Zerwas and P.M. Zerwas, Exploring the Higgs portal, Phys. Lett. B 703 (2011) 298 [arXiv:1106.3097] [InSPIRE].

[73] C. Englert, T. Plehn, M. Rauch, D. Zerwas and P.M. Zerwas, LHC: standard Higgs and hidden Higgs, Phys. Lett. B 707 (2012) 512 [arXiv:1112.3007] [INSPIRE].

[74] A. Djouadi, O. Lebedev, Y. Mambrini and J. Quevillon, Implications of LHC searches for Higgs-portal dark matter, Phys. Lett. B 709 (2012) 65 [arXiv:1112.3299] [INSPIRE].

[75] O. Lebedev, On stability of the electroweak vacuum and the Higgs portal, Eur. Phys. J. C 72 (2012) 2058 [arXiv: 1203.0156] [INSPIRE].

[76] K. Cranmer, S. Kreiss, D. Lopez-Val and T. Plehn, Decoupling theoretical uncertainties from measurements of the Higgs boson, Phys. Rev. D 91 (2015) 054032 [arXiv:1401.0080] [INSPIRE].

[77] K. Agashe, R. Contino and A. Pomarol, The minimal composite Higgs model, Nucl. Phys. B 719 (2005) 165 [hep-ph/0412089] [INSPIRE].

[78] F. Bonnet, M.B. Gavela, T. Ota and W. Winter, Anomalous Higgs couplings at the LHC and their theoretical interpretation, Phys. Rev. D 85 (2012) 035016 [arXiv:1105.5140] [INSPIRE].

[79] F. Bonnet, T. Ota, M. Rauch and W. Winter, Interpretation of precision tests in the Higgs sector in terms of physics beyond the standard model, Phys. Rev. D 86 (2012) 093014 [arXiv:1207.4599] [INSPIRE].

[80] V. Drollinger, T. Müller and D. Denegri, Searching for Higgs bosons in association with top quark pairs in the $H^{0} \rightarrow b \bar{b}$ decay mode, hep-ph/0111312 [INSPIRE].

[81] J. Cammin and M. Schumacher, The ATLAS discovery potential for the channel $t \bar{t} H$, $H \rightarrow b \bar{b}$, ATL-PHYS-2003-024, CERN, Geneva Switzerland (2003).

[82] T. Plehn, G.P. Salam and M. Spannowsky, Fat jets for a light Higgs, Phys. Rev. Lett. 104 (2010) 111801 [arXiv:0910.5472] [INSPIRE].

[83] P. Artoisenet, P. de Aquino, F. Maltoni and O. Mattelaer, Unravelling tēth via the matrix element method, Phys. Rev. Lett. 111 (2013) 091802 [arXiv: 1304.6414] [INSPIRE].

[84] D. Curtin, J. Galloway and J.G. Wacker, Measuring the țth coupling from same-sign dilepton $+2 b$ measurements, Phys. Rev. D 88 (2013) 093006 [arXiv:1306.5695] [InSPIRE].

[85] P. Agrawal, S. Bandyopadhyay and S.P. Das, Multilepton signatures of the Higgs boson through its production in association with a top-quark pair, Phys. Rev. D 88 (2013) 093008 [arXiv: 1308.3043] [INSPIRE].

[86] M.R. Buckley, T. Plehn, T. Schell and M. Takeuchi, Buckets of Higgs and tops, JHEP 02 (2014) 130 [arXiv:1310.6034] [INSPIRE]. 
[87] M. Buschmann et al., Mass effects in the Higgs-gluon coupling: boosted vs off-shell production, JHEP 02 (2015) 038 [arXiv:1410.5806] [INSPIRE].

[88] O.J.P. Eboli and D. Zeppenfeld, Observing an invisible Higgs boson, Phys. Lett. B 495 (2000) 147 [hep-ph/0009158] [INSPIRE].

[89] C. Bernaciak, T. Plehn, P. Schichtel and J. Tattersall, Spying an invisible Higgs boson, Phys. Rev. D 91 (2015) 035024 [arXiv:1411.7699] [InSPIRE].

[90] R.M. Godbole, M. Guchait, K. Mazumdar, S. Moretti and D.P. Roy, Search for 'invisible' Higgs signals at LHC via associated production with gauge bosons, Phys. Lett. B 571 (2003) 184 [hep-ph/0304137] [INSPIRE].

[91] A. Hocker, H. Lacker, S. Laplace and F. Le Diberder, A new approach to a global fit of the CKM matrix, Eur. Phys. J. C 21 (2001) 225 [hep-ph/0104062] [INSPIRE].

[92] M. Cacciari and N. Houdeau, Meaningful characterisation of perturbative theoretical uncertainties, JHEP 09 (2011) 039 [arXiv:1105.5152] [INSPIRE].

[93] E. Bagnaschi, M. Cacciari, A. Guffanti and L. Jenniches, An extensive survey of the estimation of uncertainties from missing higher orders in perturbative calculations, JHEP 02 (2015) 133 [arXiv: 1409.5036] [INSPIRE].

[94] T. Plehn, Lectures on LHC physics, http://www.thphys.uni-heidelberg.de/ plehn/pics/lhc.pdf, Germany (2015) [Lect. Notes Phys. 844 (2012) 1] [arXiv: 0910.4182] [INSPIRE].

[95] A. David and G. Passarino, How well can we guess theoretical uncertainties?, Phys. Lett. B 726 (2013) 266 [arXiv:1307.1843] [INSPIRE].

[96] C. Anastasiou, C. Duhr, F. Dulat, F. Herzog and B. Mistlberger, Higgs boson gluon-fusion production in QCD at three loops, Phys. Rev. Lett. 114 (2015) 212001 [arXiv:1503.06056] [INSPIRE].

[97] LHC Higgs Cross section Working Group collaboration, S. Dittmaier et al., Handbook of LHC Higgs cross sections: 1. Inclusive observables, CERN-2011-002, CERN, Geneva Switzerland (2011) [arXiv: 1101.0593] [INSPIRE].

[98] LHC Higgs Cross section Working Group collaboration, S. Dittmaier et al., Handbook of LHC Higgs cross sections: 2. Differential distributions, CERN-2012-002, CERN, Geneva Switzerland (2012) [arXiv:1201.3084] [INSPIRE].

[99] LHC Higgs Cross section Working Group collaboration, J.R. Andersen et al., Handbook of LHC Higgs cross sections: 3. Higgs properties, CERN-2013-004, CERN, Geneva Switzerland (2013) [arXiv: 1307.1347] [INSPIRE].

[100] LHC CrossSections webpage, https://twiki.cern.ch/twiki/bin/view/LHCPhysics/CrossSections.

[101] S. Weinberg, Phenomenological Lagrangians, Physica 96A (1979) 327 [InSPIRE].

[102] H. Georgi, Weak interactions and modern particle theory, Benjamim/Cummings, Menlo Park U.S.A. (1984).

[103] J.F. Donoghue, E. Golowich and B.R. Holstein, Dynamics of the standard model, Cambridge University Press, Cambridge U.K. (1992) [INSPIRE].

[104] C.N. Leung, S.T. Love and S. Rao, Low-energy manifestations of a new interaction scale: operator analysis, Z. Phys. C 31 (1986) 433 [INSPIRE]. 
[105] W. Buchmüller and D. Wyler, Effective Lagrangian analysis of new interactions and flavor conservation, Nucl. Phys. B 268 (1986) 621 [INSPIRE].

[106] A. De Rujula, M.B. Gavela, P. Hernández and E. Masso, The selfcouplings of vector bosons: does LEP-1 obviate LEP-2?, Nucl. Phys. B 384 (1992) 3 [INSPIRE].

[107] K. Hagiwara, R. Szalapski and D. Zeppenfeld, Anomalous Higgs boson production and decay, Phys. Lett. B 318 (1993) 155 [hep-ph/9308347] [INSPIRE].

[108] K. Hagiwara, S. Matsumoto and R. Szalapski, Constraints on new physics in the electroweak bosonic sector from current data and future experiments, Phys. Lett. B 357 (1995) 411 [hep-ph/9505322] [INSPIRE].

[109] M.C. Gonzalez-Garcia, Anomalous Higgs couplings, Int. J. Mod. Phys. A 14 (1999) 3121 [hep-ph/9902321] [INSPIRE].

[110] G. Passarino, NLO inspired effective Lagrangians for Higgs physics, Nucl. Phys. B 868 (2013) 416 [arXiv : 1209.5538] [inSPIRE].

[111] K. Hagiwara, S. Ishihara, R. Szalapski and D. Zeppenfeld, Low-energy effects of new interactions in the electroweak boson sector, Phys. Rev. D 48 (1993) 2182 [InSPIRE].

[112] K. Hagiwara, T. Hatsukano, S. Ishihara and R. Szalapski, Probing nonstandard bosonic interactions via $W$ boson pair production at lepton colliders, Nucl. Phys. B 496 (1997) 66 [hep-ph/9612268] [INSPIRE].

[113] B. Grzadkowski, M. Iskrzynski, M. Misiak and J. Rosiek, Dimension-6 terms in the standard model Lagrangian, JHEP 10 (2010) 085 [arXiv: 1008.4884] [INSPIRE].

[114] T. Appelquist and J. Carazzone, Infrared singularities and massive fields, Phys. Rev. D 11 (1975) 2856 [INSPIRE].

[115] C. Englert et al., Precision measurements of Higgs couplings: implications for new physics scales, J. Phys. G 41 (2014) 113001 [arXiv:1403.7191] [inSPIRE].

[116] A. Thamm, Effective Lagrangian perspectives on electroweak symmetry breaking, Ph.D. thesis, http://infoscience.epfl.ch/record/201641, EPFL, Lausanne Switzerland (2014) [INSPIRE].

[117] A. Falkowski, Effective field theory approach to LHC Higgs data, arXiv:1505.00046 [INSPIRE].

[118] J. Alwall, M. Herquet, F. Maltoni, O. Mattelaer and T. Stelzer, MadGraph 5: going beyond, JHEP 06 (2011) 128 [arXiv:1106.0522] [INSPIRE].

[119] N.D. Christensen and C. Duhr, FeynRules - Feynman rules made easy, Comput. Phys. Commun. 180 (2009) 1614 [arXiv:0806.4194] [InSPIRE].

[120] A. Pukhov et al., CompHEP: a package for evaluation of Feynman diagrams and integration over multiparticle phase space, hep-ph/9908288 [INSPIRE].

[121] CompHEP collaboration, E. Boos et al., CompHEP 4.4: automatic computations from Lagrangians to events, Nucl. Instrum. Meth. A 534 (2004) 250 [hep-ph/0403113] [INSPIRE].

[122] K. Arnold et al., VBFNLO: a parton level Monte Carlo for processes with electroweak bosons, Comput. Phys. Commun. 180 (2009) 1661 [arXiv:0811.4559] [INSPIRE].

[123] J. Baglio et al., Release note - VBFNLO 2.7.0, arXiv:1404.3940 [InSPIRE]. 
[124] J. Frank, M. Rauch and D. Zeppenfeld, Spin-2 resonances in vector-boson-fusion processes at next-to-leading order QCD, Phys. Rev. D 87 (2013) 055020 [arXiv:1211.3658] [INSPIRE].

[125] J. Frank, M. Rauch and D. Zeppenfeld, Higgs spin determination in the $W W$ channel and beyond, Eur. Phys. J. C 74 (2014) 2918 [arXiv:1305.1883] [inSPIRE].

[126] C. Englert, D. Goncalves-Netto, K. Mawatari and T. Plehn, Higgs quantum numbers in weak boson fusion, JHEP 01 (2013) 148 [arXiv:1212.0843] [INSPIRE].

[127] A. Djouadi, R.M. Godbole, B. Mellado and K. Mohan, Probing the spin-parity of the Higgs boson via jet kinematics in vector boson fusion, Phys. Lett. B 723 (2013) 307 [arXiv: 1301.4965] [INSPIRE].

[128] P. Artoisenet et al., A framework for Higgs characterisation, JHEP 11 (2013) 043 [arXiv: 1306.6464] [INSPIRE].

[129] T. Sjöstrand, S. Mrenna and P.Z. Skands, PYTHIA 6.4 physics and manual, JHEP 05 (2006) 026 [hep-ph/0603175] [INSPIRE].

[130] J. Conway, PGS4 Pretty Good Simulation of high energy collisions webpage, http://www.physics.ucdavis.edu/ conway/research/software/pgs/pgs4-general.htm, (2012).

[131] DELPHES 3 collaboration, J. de Favereau et al., DELPHES 3, a modular framework for fast simulation of a generic collider experiment, JHEP 02 (2014) 057 [arXiv:1307.6346] [INSPIRE].

[132] T. Plehn, D.L. Rainwater and D. Zeppenfeld, Determining the structure of Higgs couplings at the LHC, Phys. Rev. Lett. 88 (2002) 051801 [hep-ph/0105325] [INSPIRE].

[133] V. Hankele, G. Klamke, D. Zeppenfeld and T. Figy, Anomalous Higgs boson couplings in vector boson fusion at the CERN LHC, Phys. Rev. D 74 (2006) 095001 [hep-ph/0609075] [INSPIRE].

[134] C. Ruwiedel, N. Wermes and M. Schumacher, Prospects for the measurement of the structure of the coupling of a Higgs boson to weak gauge bosons in weak boson fusion with the ATLAS detector, Eur. Phys. J. C 51 (2007) 385 [inSPIRE].

[135] K. Hagiwara, Q. Li and K. Mawatari, Jet angular correlation in vector-boson fusion processes at hadron colliders, JHEP 07 (2009) 101 [arXiv:0905.4314] [INSPIRE].

[136] A. Djouadi, R.M. Godbole, B. Mellado and K. Mohan, Probing the spin-parity of the Higgs boson via jet kinematics in vector boson fusion, Phys. Lett. B 723 (2013) 307 [arXiv:1301.4965] [INSPIRE].

[137] The Durham HepData project webpage, http://hepdata.cedar.ac.uk/.

[138] A. Biekötter, A. Knochel, M. Krämer, D. Liu and F. Riva, Vices and virtues of Higgs effective field theories at large energy, Phys. Rev. D 91 (2015) 055029 [arXiv:1406.7320] [INSPIRE].

[139] M. Gorbahn, J.M. No and V. Sanz, Benchmarks for Higgs effective theory: extended Higgs sectors, arXiv:1502.07352 [INSPIRE].

[140] A. Drozd, J. Ellis, J. Quevillon and T. You, Comparing EFT and exact one-loop analyses of non-degenerate stops, JHEP 06 (2015) 028 [arXiv: 1504.02409] [INSPIRE]. 
[141] A. Azatov, C. Grojean, A. Paul and E. Salvioni, Taming the off-shell Higgs boson, Zh. Eksp. Teor. Fiz. 147 (2015) 410 [J. Exp. Theor. Phys. 120 (2015) 354] [arXiv:1406.6338] [INSPIRE].

[142] J. Brehmer, J. Jaeckel and T. Plehn, Polarized $W W$ scattering on the Higgs pole, Phys. Rev. D 90 (2014) 054023 [arXiv: 1404.5951] [InSPIRE].

[143] J.M. Campbell, R.K. Ellis and C. Williams, MCFM - Monte Carlo for FeMtobarn processes webpage, http://mcfm.fnal.gov.

[144] M. Bonvini, F. Caola, S. Forte, K. Melnikov and G. Ridolfi, Signal-background interference effects for $g g \rightarrow H \rightarrow W^{+} W^{-}$beyond leading order, Phys. Rev. D 88 (2013) 034032 [arXiv: 1304.3053] [INSPIRE].

[145] A. Banfi, A. Martin and V. Sanz, Probing top-partners in Higgs+jets, JHEP 08 (2014) 053 [arXiv:1308.4771] [INSPIRE].

[146] A. Azatov and A. Paul, Probing Higgs couplings with high $p_{T}$ Higgs production, JHEP 01 (2014) 014 [arXiv: 1309.5273] [INSPIRE].

[147] C. Englert, M. McCullough and M. Spannowsky, Gluon-initiated associated production boosts Higgs physics, Phys. Rev. D 89 (2014) 013013 [arXiv: 1310.4828] [INSPIRE].

[148] C. Grojean, E. Salvioni, M. Schlaffer and A. Weiler, Very boosted Higgs in gluon fusion, JHEP 05 (2014) 022 [arXiv: 1312.3317] [InSPIRE].

[149] M. Buschmann, C. Englert, D. Goncalves, T. Plehn and M. Spannowsky, Resolving the Higgs-gluon coupling with jets, Phys. Rev. D 90 (2014) 013010 [arXiv:1405.7651] [INSPIRE].

[150] B. Hespel, F. Maltoni and E. Vryonidou, Higgs and $Z$ boson associated production via gluon fusion in the SM and the $2 H D M$, JHEP 06 (2015) 065 [arXiv:1503.01656] [INSPIRE]. 